\title{
GENERAL SUPERSTRUCTURE AND GLOBAL OPTIMIZATION FOR THE DESIGN OF INTEGRATED PROCESS WATER NETWORKS
}

\author{
Elvis Ahmetović ${ }^{1}$, Ignacio E. Grossmann ${ }^{2, *}$ \\ ${ }^{1}$ Department of Process Engineering, University of Tuzla, 75000 Tuzla, Bosnia and Herzegovina \\ ${ }^{2}$ Department of Chemical Engineering, Carnegie Mellon University, Pittsburgh, PA 15213, United States
}

\begin{abstract}
In this paper, we propose a general superstructure and a model for the global optimization for the design of integrated process water networks. The superstructure consists of multiple sources of water, water-using processes, wastewater treatment and pre-treatment operations. The unique features are first, that all feasible interconnections are considered between them, including water re-use, water regeneration and re-use, water regeneration recycling, local recycling around process and treatment units. Second, multiple sources of water of different quality that can be used in the various operations are included. Third, the superstructure incorporates both mass transfer and non-mass transfer operations. The proposed model of the integrated water network is formulated as a Nonlinear Programming (NLP) and as a Mixed Integer Nonlinear Programming (MINLP) problem for the case when 0-1 variables are included to model the cost of piping and/or selection of technologies for treatment. The MINLP model can be used to find optimal network designs with different number of streams in the piping network. In this work, we propose to represent the bounds on the variables as general equations obtained by physical inspection of the superstructure and using logic specifications needed for solving the model. We also incorporate the cut proposed by Karuppiah and Grossmann (2006) to significantly improve the strength of the lower bound for the global optimum. The proposed model is tested on the several illustrative examples, including large-scale problems.
\end{abstract}

Keywords: Integrated water networks; Superstructure optimization; Nonconvex NLP and MINLP model.

\section{Introduction}

The process industry consumes a large amount of water. For instance, water is used for washing operations, separation processes, steam and power generation, cooling, etc. These processes in turn generate wastewater, which is usually processed in treatment units before discharge to the environment. The shortage of freshwater, its increasing cost and the one of treatment processes, as well as strict environmental regulations on the industrial effluents, provide a strong motivation for developing approaches and techniques to design more efficient process water networks.

\footnotetext{
* Corresponding author. Tel.: +1 412268 2230; fax: +1 4122687139 .

E-mail address: grossmann@ @ cmu.edu (I.E. Grossmann).
} 
The two major approaches for the optimal design of water network systems are water pinch technology and mathematical programming. A comprehensive review of these approaches as well as systematic methods of chemical process design are given by Rossiter (1995), El-Halwagi (1997), Biegler, Grossmann and Westerberg (1997), Mann and Liu (1999), Bagajewicz (2000), Ježowski (2008), Bagajewicz and Faria (2009), and Foo (2009).

Water pinch technology relies on graphic representations and it is based on an extension of pinch analysis technique for heat integration (Linnhoff and Hindmarsh 1983). The first authors, who introduced the notion of synthesizing mass-exchange networks (MEN's), were El-Halwagi and Manousiouthakis $(1989 ; 1990)$. They considered mass exchange between the rich and the lean process streams. After that, a targeting approach for minimum freshwater consumption was developed by Wang and Smith (1994a; 1994b; 1995) and later extended and improved by a number of researchers (Dhole, Ramchandani, Tainsh \& Wasilewski, 1996; Doyle \& Smith, 1997; Kuo \& Smith, 1997; Castro, Matos, Fernandes \& Nunes, 1999; Sorin \& Bedard, 1999; Polley \& Polley, 2000; Hallale, 2002; Bandyopadhyay, Ghanekar \& Pillai, 2006; Foo, Kazantzi, El-Halwagi \& Abdul Manan, 2006; Foo, 2009).

The mathematical programming approach is based on the optimization of a superstructure. The seminal paper addressed a mathematical programming formulation of water network was given by Takama, Kuriyama, Shiroko \& Umeda (1980). They considered a total system consisting of waterusing and wastewater-treating units. In addition to this, they generated a superstructure of all possible re-use and regeneration opportunities and formulated the problem of optimal water allocation in a petroleum refinery as a nonlinear programming problem. After their paper the solution of the mathematical programming formulation for this problem was not addressed for many years (Bagajewicz, 2000). In many papers, the total water network is decomposed into two parts (network with water-using operations and wastewater treatment network) which are solved separately. For example, Kuo and Smith (1997) presented an extension of the methodology for the design of distributed effluent treatment systems previously given by Wang and Smith (1994b). They presented an improved method for targeting the treatment flowrate and the distribution of load between multiple treatment processes. In addition to this, Galan and Grossmann (1998) addressed the optimal design of distributed wastewater network where multiple contaminants are considered. They proposed a heuristic search procedure based on the successive solution of a relaxed linear model and the original nonconvex nonlinear model. Their procedure has the capability of finding global or near global optimum solutions. In addition, the model was extended for selecting different treatment technologies 
for handling membrane separation modules. Savelski and Bagajewicz (2000; 2003) developed necessary optimality conditions (maximum outlet concentrations from water-using units and concentration monotonicy) for single and multiple water allocation systems in refineries and process plants. They used these conditions to eliminate the nonlinearities in the water network models arising in the mass balance equations in the form of bilinear terms (concetration times flowrate). According to this, they showed that the nonlinear model of water networks for single component can be linearized. Quesada and Grossmann (1995) proposed a rigorous procedure for the global optimization of bilinear process networks with multicomponent streams. Their procedure is based on a reformulation-linearization technique applied to nonlinear models in order to obtain a relaxed linear programming formulation that provides a valid lower bound to the global optimum. Castro, Teles and Novais (2009) proposed the two-stage solution strategy for the optimal design of distributed wastewater networks with multiple contaminants. In the first stage, a decomposition method is employed that replaces the nonlinear program by a succession of linear programs, one for each treatment unit. In the second, stage, the resulting network is used as a starting point for the solution of the nonlinear model with a local optimization solver.

The problem of designing the total water networks has been addressed in relatively few papers (Ježovski, 2008). Doyle and Smith (1997) proposed a method based on nonlinear programming for targeting maximum water reuse in processing systems. To overcome the difficulties associated with the nonlinear optimization model, they used a linear model to provide an initialization for the nonlinear model. Alva-Argáez, Kokossis and Smith (1998) proposed an integrated methodology for the design of industrial water systems. Their decomposition strategy is based on a recursive procedure where the original Mixed-Integer Nonlinear Problem (MINLP) problem is replaced by a sequence of Mixed-Integer Linear Problems (MILPs). Huang, Chang, Ling, and Chang (1999) proposed a mathematical model for determining the optimal water usage and treatment network in any chemical plant. They presented a modified version of the superstructure proposed by Takama, Kuriyama, Shiroko, and Umeda (1980) and included in the model design equations of all wastewater treatment facilities and all units which utilize either process or utility water so that better integration on a planwide scale can be achieved. Feng and Seider (2001) proposed a network structure in which internal water mains are utilized. Their structure simplifies the piping network as well as the operation and control of large plants involving many water-using processes. Gunaratnam, Alva-Argaez, Kokossis, Kim, and Smith (2005) presented an automated design of total water systems where the optimal distribution of water to satisfy process demands and optimal treatment of effluent streams are 
considered simultaneously. They used a two-stage optimization approach to solve the MINLP model involving an MILP in the first stage to initialize the problem. In the second stage, the design is finetuned using MINLP. In addition to this, the network complexity is controlled by specifying the minimum permissible flowrates in the network. Their methodology provides a robust technique but it does necessarily yield the global optimum.

Karuppiah and Grossmann (2006) addressed the problem of optimal synthesis of an integrated water system consisting of water-using processes and water treatment operations. In contrast to previous work, they proposed a spatial branch and contract algorithm for the rigorous global optimization of the nonlinear program of the integrated water system. In their algorithm, tight lower bounds on the global optimum are obtained by solving a relaxation of the original problem obtained by approximating the nonconvex terms in the NLP model with piecewise linear estimators. Li and Chang (2007) developed an efficient initialization strategy to solve the NLP and MINLP models for water network with multiple contaminants. In the MINLP model they formulated structural constraints to manipulate structural complexity, but global optimality is not guaranteed. Also, they reported that the optimum solution obtained by the initialization strategy is at least as good as results reported in the literature but with less computation time to achieve convergence. In the same year, Alva-Argaez, Kokossis, and Smith (2007) proposed a systematic approach to address water reuse in oil refineries. The methodology is based on the water-pinch decomposition. Their approach simplifies the challenges of the optimization problem making systematic use of water-pinch insights to define successive projections in the solution space.

Putra and Amminudin (2008) proposed an alternative solution strategy for solving the total water system design problem by utilizing the MILP and NLP in a two-step optimization approach. Their approach, which is not guaranteed to find the global optimum, has the capability of generating multiple optimal solutions and to handle practical considerations, as well as to provide users with the ability to control water network during the optimization process. Luo and Uan (2008) presented a superstructure-based method for the optimization of integrated water systems with the heuristic Particle Swarm Optimization (PSO) algorithm. Karuppiah and Grossmann (2008) presented a formulation for representing and optimizing integrated water networks operating under uncertain conditions of the contaminant loads in the process units and contaminant removals of the treatment units. They formulated a multi-scenario nonconvex MINLP model for globally optimizing an integrated water network operating under uncertainty. Further, they proposed a spatial branch and cut 
algorithm combining the concepts of Lagrangean relaxation and convex relaxation in order to generate strong bounds for the global optimum.

Notice that in the previously mentioned papers the vast majority are based on linearization of the nonlinear models, or use linear models to provide an initialization for the nonlinear models, which are solved with local optimization solvers. Moreover, a number of authors replace the MINLP problems by a sequence of the MILPs. Bagajewicz and Faria (2009) and Faria and Bagajewicz (2009) presented the evolution of the water network allocation problem given by Takama, Kuriyama, Shiroko \& Umeda (1980). In addition, they included the water pre-treatment subsystem in the network and discussed the end-of-pipe wastewater treatment and the complete water integration.

It should be mentioned that the problem of designing total water networks has been addressed in relatively few papers due to its complexity (Ježovski, 2008). The main complexity in the nonlinear model appears due to the bilinear terms in the mass balance equations (flowrate times concentration) and the concave cost terms in the objective function with which the solution for the total water network is not guaranteed to be the global optimum. In addition to this, it is worth pointing out that in the majority of the published papers all possible options are not considered in the total water network such as all feasible interconnections in the network, multiple sources of water of different quality, pre-treatment of the water, mass transfer and non-mass transfer water-using operations. Moreover, in many papers the cost of water pumping through pipes and the investment cost for pipes are not included in the objective function.

The main objective of this paper is to propose a general superstructure and a global optimization approach for the design of integrated process water networks. The superstructure consists of multiple sources of water, water-using (mass transfer and non-mass transfer) processes, water treatment operations and all feasible interconnections in the network. We propose nonconvex NLP and MINLP models for the integrated water network. We also develop bounds on the variables as general equations obtained by physical inspection of the superstructure and use logic specifications that have the capability of reducing the feasible region, and helping global the NLP and MINLP solvers to find more efficiently the global optimum. In the MINLP model we include the costs of piping and the costs of water pumping through pipes. With the proposed model we can also control the complexity of the piping network. For large-scale industrial MINLP problems, we propose a two-stage solution method in which we first solve the NLP model in order to fix a subset of 0-1 variables to zero so as to solve a reduced size MINLP. We also update the bounds to tighten the reduced MINLP model. Several examples, including large-scale industrial problems, are presented in the paper to illustrate 
the proposed method. We use the general purpose global optimization solvers BARON (Sahinidis, 1996) and LINDOGlobal to solve the proposed models to global optimality.

The outline of the paper is as follows. First, we present the problem statement. We then introduce the general water network superstructure, followed by its mathematical formulation. Further, we present two-stage solution methods for solving large-scale industrial MINLP problems. The computational results and discussion are given in the following section. Finally, in the last section we present general conclusions.

\section{Problem statement}

The problem addressed in this paper can be stated as follows. Given is a set of single/multiple water sources with/without contaminants, a set of water-using units and wastewater treatment operations, fixed water demands of process units, maximum concentrations of contaminants in inlet streams at process units, mass loads of contaminants in process units, the costs of water sources and wastewater treatment units, \% removal for each contaminant in treatment units and the maximum contaminant concentrations in the discharge effluent to the environment. The problem consists in determining the interconnections, flowrates and contaminants concentration of each stream in the water network, the freshwater consumption and wastewater generation, and the total annual cost of the water network (costs of freshwater consumption, wastewater treatment, piping network, pumping water through pipes in the network).

The proposed model is based on the following assumptions: the number of water sources is fixed, the number of water-using and water treatment operations is fixed, the flowrates through the waterusing wastewater treatment operations are fixed, the network operates under isothermal condition and isobaric conditions.

\section{Superstructure of the integrated process water network}

The proposed model of the integrated water network relies on the superstructure given in Figure 1. The superstructure, which is an extension and generalization of the one given by Karuppiah and Grossmann (2006), consists of one or multiple sources of water of different quality, water-using processes, and wastewater treatment operations. The unique feature is that all feasible connections are considered between them, including water re-use, water regeneration and re-use, water regeneration recycling, local recycling around process and treatment units and pre-treatment of feedwater streams. Multiple sources of water include water of different quality that can be used in the various operations, 
and which may be sent first for pre-treatment. The superstructure incorporates both the mass transfer and non-mass transfer operations. According to this, it can be used to represent separate subsystems as well as an integrated total system. Furthermore, it enables modeling different types of water network optimization problems as will be shown later in the paper.

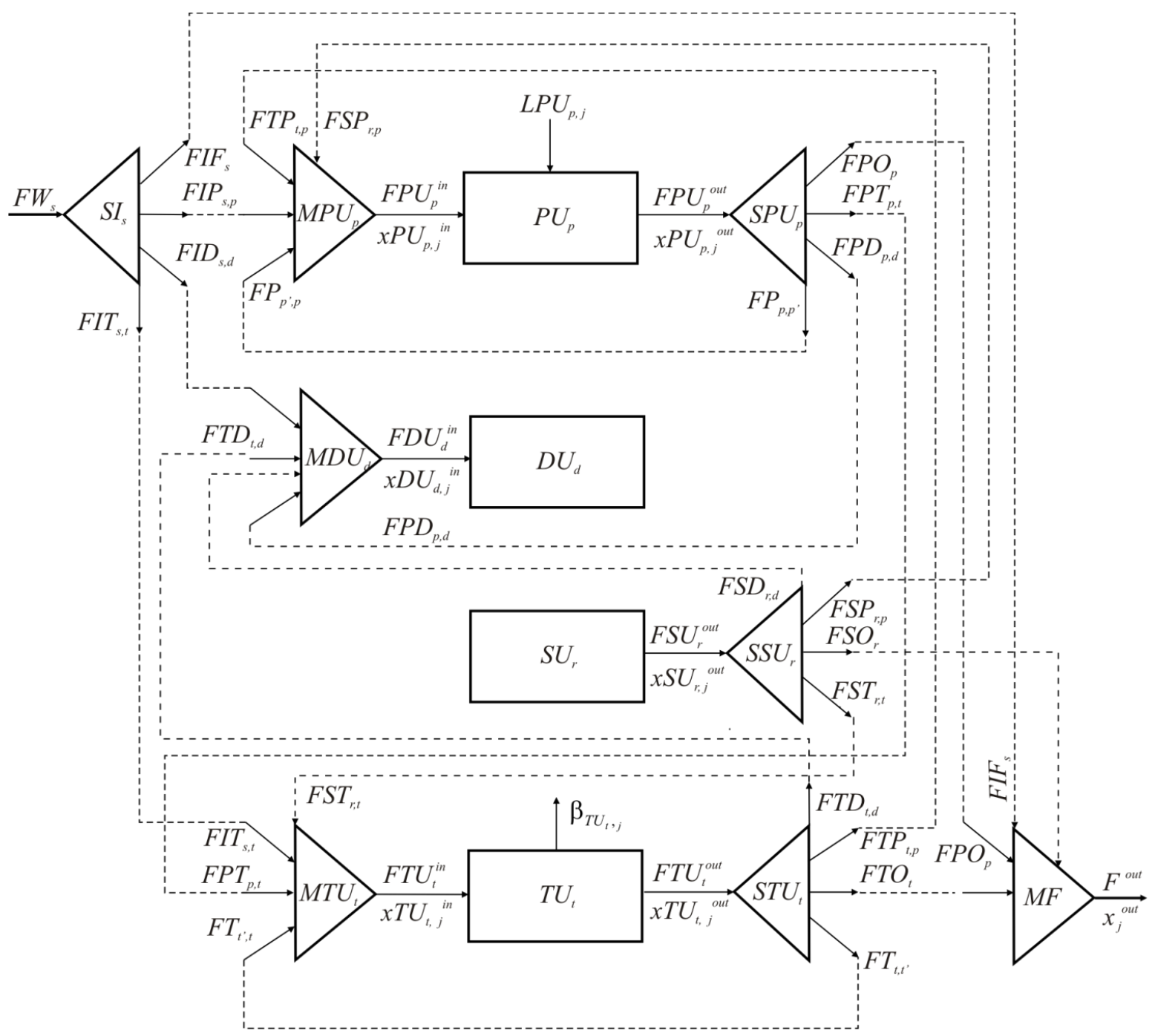

Figure 1. Generalized superstructure for the design of integrated process water networks.

\section{Basic conceptual options of models}

The mathematical model of integrated process water networks consists of mass balance equations for water and the contaminants for every unit in the network. The model is formulated as a nonconvex nonlinear programming (NLP), and as a nonconvex mixed-integer nonlinear programming (MINLP) for the case when 0-1 variables are included to model the cost of piping and/or selection of technologies for treatment. The nonlinearities in the models appear in the mass balance equations in 
the form of bilinear terms (concentration times flowrate). In addition to this, nonlinearities appear in the objective function as concave terms of the cost functions for the water-treatment operations.

Hence, the water network models are nonconvex, and in most cases lead to multiple local solutions making difficult to obtain the global or a near-global optimal solution. The basic options of the proposed water network optimization model are shown in Figure 2.

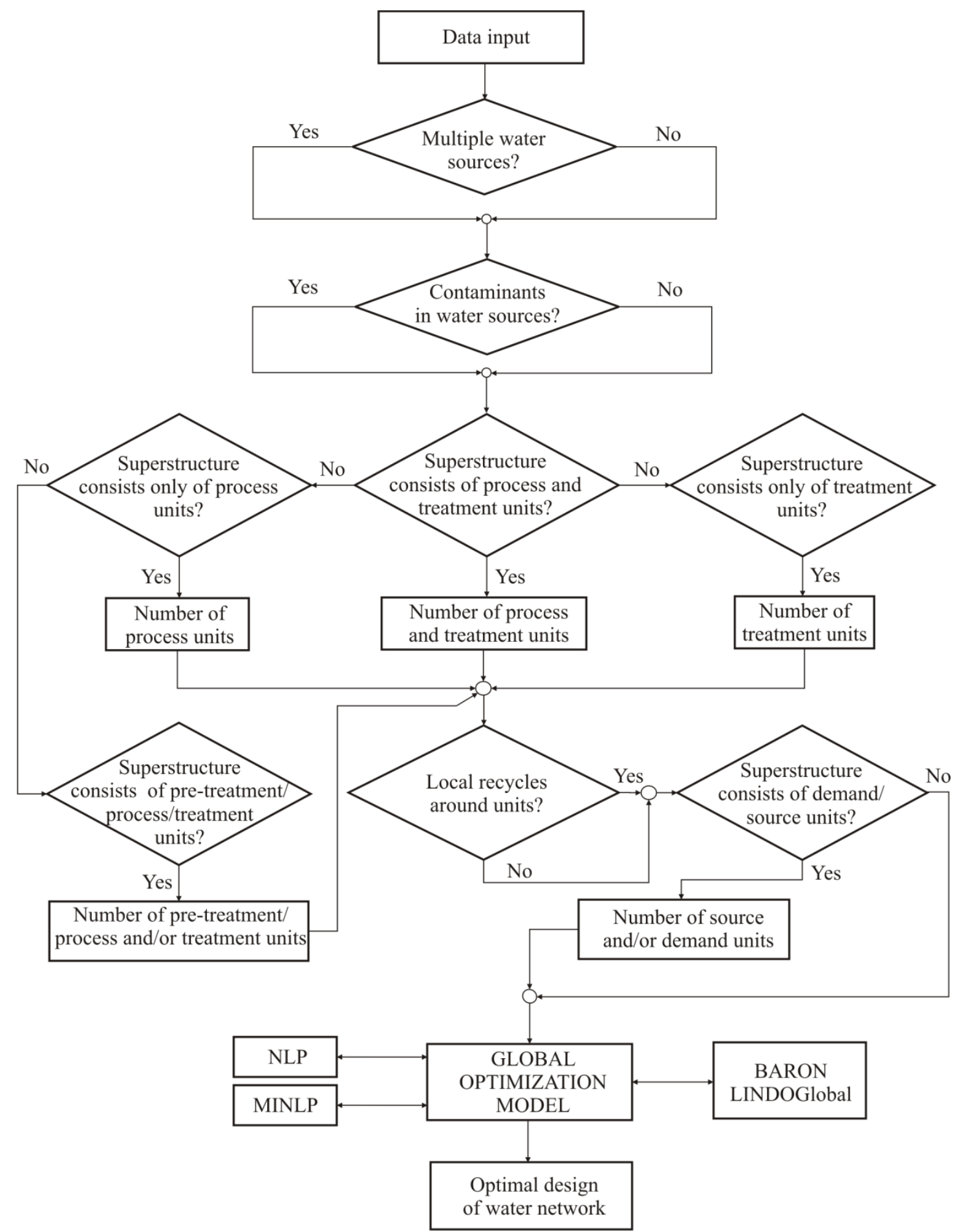

Figure 2. The basic options of the proposed optimization model. 
First of all, the model enables the choice of a single or multiple sources of water that are available for the network plant operation. Second, water sources can be clean water without contaminants or water with multiple contaminants. Water with higher quality is more expensive and the total cost will be minimized at the expense of lower quality water as shown later in illustrative examples. Water with contaminants on the other hand may be directly sent to treatment units before using it for waterusing operations. Third, the water network can consist only of the water-using operations or wastewater treatment operations. In addition, it can be an integrated water network including both water-using operations and wastewater treatment operations. Fourth, all feasible interconnections are possible in the network for every option mentioned before. Fifth, it is possible to choose local recycles around the water-using operations or the wastewater treatment operations. Local recycling (Wang \& Smith, 1995) can be used to satisfy the flowrate constraints and in these cases it is possible to have an additional reduction in water consumption as is shown later in an illustrative example. Sixth, as industrial water network systems usually consist of different types of water-using operations that can be classified as mass-transfer operations and non-mass transfer operations (Mann \& Liu, 1999), both types of these operations are included in the superstructure. In addition to this, in many processes there is loss of water that is not available for re-use in a water-using operation. Hence, this unit involves water demand unit and is a water sink. Moreover, from some water-using operations water is available for re-use in other operations and they represent sources of water. In all the above mentioned options, the number of contaminants, water sources, water-using operations and wastewater treatment operations is fixed. The proposed model enables modeling and optimization of any of the above mentioned options of the water network superstructure.

\subsection{Mathematical model}

In this section we propose an MINLP model for the superstructure given in Figure 1.

\section{Nomenclature}

Sets and Indices

j contaminant

DU $\quad$ set of demand units

$d \quad$ demand unit

$P U \quad$ set of process units

$p \quad$ process unit 


$\begin{array}{ll}S U & \text { set of source units } \\ r & \text { source unit } \\ S W & \text { set of freshwater sources } \\ S & \text { freshwater source } \\ T U & \text { set of treatment units } \\ t & \text { treatment unit }\end{array}$

\section{Parameters}

$A R \quad$ annualized factor for investment for treatment units

$C F W_{s} \quad$ cost of freshwater from source $s$

$C P \quad$ fixed cost for each individual pipe in the network

$F D U_{d}^{i n} \quad$ mass flowrate of inlet water stream in demand unit $d$

$F P U_{p}^{i n} \quad$ mass flowrate of inlet water stream in process unit $p$

$F S U_{r}^{\text {out }} \quad$ mass flowrate of outlet water stream from source unit $r$

$F W W_{s} \quad$ mass flowrate of freshwater source $s$

$H \quad$ hours of plant operation per annum

$I C_{t} \quad$ investment cost coefficient for treatment unit $t$

IP variable cost for each individual pipe in the network

$L P U_{p, j} \quad$ load of contaminant $j$ in process unit $p$

$N_{\max } \quad$ maximum number of streams for the network

$O C_{t} \quad$ operating cost coefficient for treatment unit $t$

$P M \quad$ operating cost coefficient for pumping water through each pipe in the network

$R_{p}, R_{t} \quad$ local recycle around process unit $p$ and treatment unit $t$ ( $R_{p}=0$ and $R_{t}=0$ does not exist, $R_{p}=1$ and $R_{t}=1$ if exists)

$R R_{t, j} \quad \%$ removal for contaminant $j$ in treatment unit $t$

$x_{j}^{\text {out,max }} \quad$ maximum concentration of contaminant $j$ in discharge stream to the environment

$x D U_{d . j}^{i n, \max } \quad$ maximum concentration of contaminant $j$ in inlet stream into demand unit $d$

$x P U_{p . j}^{i n, \max } \quad$ maximum concentration of contaminant $j$ in inlet stream into process unit $p$

$x S U_{r . j}^{\text {out }} \quad$ concentration of contaminant $j$ in outlet stream from source unit $r$ 


$$
\begin{array}{ll}
x W_{s . j}^{\text {in }} & \text { concentration of contaminant } j \text { in freshwater source } s \\
\alpha & \text { cost exponent for treatment units }(0 \leq \alpha \leq 1) \\
\beta_{T U t, j} & 1-\left(R R_{t, j} / 100\right) \\
\gamma & \text { cost exponent for pipes }(0 \leq \gamma \leq 1)
\end{array}
$$

\section{Continuous variables}

$F^{\text {out }} \quad$ mass flowrate of outlet wastewater stream from final mixer

$F I D_{s, d} \quad$ mass flowrate of water stream from freshwater source $s$ to demand unit $d$

$F_{s} \quad$ mass flowrate of water stream from freshwater source $s$ to final mixer

$F I P_{s, p} \quad$ mass flowrate of water stream from freshwater source $s$ to process unit $p$

$F I T_{s, t} \quad$ mass flowrate of water stream from freshwater source $s$ to treatment unit $t$

$F P_{p^{\prime}, p} \quad$ mass flowrate of water stream from process unit $p^{\prime}$ to process unit $p$

$F P D_{p, d} \quad$ mass flowrate of water stream from process unit $p$ to demand unit $d$

$\mathrm{FPO}_{p} \quad$ mass flowrate of water stream from process unit $p$ to final mixer

$F P T_{p, t} \quad$ mass flowrate of water stream from process unit $p$ to treatment unit $t$

$F P U_{p}^{\text {out }} \quad$ mass flowrate of outlet water stream from process unit $p$

$F S D_{r, d} \quad$ mass flowrate of water stream from source unit $r$ to demand unit $d$

$\mathrm{FSO}_{r} \quad$ mass flowrate of water stream from source unit $r$ to final mixer

$F S P_{r, p} \quad$ mass flowrate of water stream from source unit $r$ to process unit $p$

$F S T_{r, t} \quad$ mass flowrate of water stream from source unit $r$ to treatment unit $t$

$F T_{t^{\prime}, t} \quad$ mass flowrate of water stream from treatment unit $t^{\prime}$ to treatment unit $t$

$F T D_{t, d} \quad$ mass flowrate of water stream from treatment unit $t$ to demand unit $d$

$\mathrm{FTO}_{t} \quad$ mass flowrate of water stream from treatment unit $t$ to final mixer

$F T P_{t, p} \quad$ mass flowrate of water stream from treatment unit $t$ to process unit $p$

$F T U_{t}^{i n} \quad$ mass flowrate of inlet water stream in treatment unit $t$

$F T U_{t}^{\text {out }} \quad$ mass flowrate of outlet water stream from treatment unit $t$

$F W_{s} \quad$ mass flowrate of water for freshwater source $s$ 
$x_{j}^{\text {out }}$

$x D U_{d, j}^{i n}$

$x P U_{p, j}^{i n}$

$x P U_{p, j}^{\text {out }}$

$x S P U_{t, j}^{\text {out }}$

$x S T U_{t, j}^{\text {out }}$

$x T U_{t, j}^{i n}$

$x T U_{t, j}^{o u t}$ concentration of contaminant $j$ in discharge stream to the environment

concentration of contaminant $j$ in inlet stream into demand unit $d$

concentration of contaminant $j$ in inlet stream into process unit $p$

concentration of contaminant $j$ in outlet stream from process unit $p$

concentration of contaminant $j$ in outlet stream from splitter process unit $p$

concentration of contaminant $j$ in outlet stream from splitter treatment unit $t$

concentration of contaminant $j$ in inlet stream into treatment unit $t$

concentration of contaminant $j$ in outlet stream from treatment unit $t$

Binary variables

$y_{F I D_{s, d}} \quad$ existence of pipe between freshwater source $s$ and demand unit $d$

$y_{F I F_{s}} \quad$ existence of pipe between freshwater source $s$ and final mixer

$y_{F I P_{s, p}} \quad$ existence of pipe between freshwater source $s$ and process unit $p$

$y_{F I T_{s, t}} \quad$ existence of pipe between freshwater source $s$ and treatment unit $t$

$y_{F P_{p^{\prime}, p}} \quad$ existence of pipe between process unit $p$ ' and process unit $p$

$y_{F P D_{p, d}} \quad$ existence of pipe between process unit $p$ and demand unit $d$

$y_{F P O_{p}} \quad$ existence of pipe between process unit $p$ and final mixer

$y_{F P T_{p, t}} \quad$ existence of pipe between process unit $p$ and treatment unit $t$

$y_{F S D_{r, d}} \quad$ existence of pipe between source unit $r$ and demand unit $d$

$y_{F S O_{r}} \quad$ existence of pipe between source unit $r$ and final mixer

$y_{F S P_{r, p}} \quad$ existence of pipe between source unit $r$ and process unit $p$

$y_{F S T_{r, t}} \quad$ existence of pipe between source unit $r$ and treatment unit $t$

$y_{F T_{t^{\prime},}} \quad$ existence of pipe between treatment unit $t^{\prime}$ and treatment unit $t$

$y_{F T D_{t, d}} \quad$ existence of pipe between treatment unit $t$ and demand unit $d$

$y_{F_{T}} \quad$ existence of pipe between treatment unit $t$ and final mixer 
$y_{F T P_{t, p}} \quad$ existence of pipe between treatment unit $t$ and process unit $p$

\section{Subscripts/superscripts}

FX fixed bound for the variable

in $\quad$ inlet stream

$L \quad$ lower bound for the variable

$\max \quad$ maximal

out outlet stream

$U \quad$ upper bound for the variable

\subsection{Initial splitters}

The feedwater of an initial splitter $S I_{s}$ (shown in Figure 3) for freshwater source $s \in S W$ can be clean water without contaminants or water with single/multiple contaminants. Freshwater from the initial splitter can be directed to the mixer process unit, the mixer treatment unit, the mixer demand units, or the final mixer. The connection between the initial splitter and the mixers in the network depends of the special network case that is considered. For instance, if we consider only multiple wastewater feeds and the wastewater distribution subsystem there will be connections between the initial splitter and the final mixer. However, in an integrated system consisting of water-using units and wastewater treatment units, connections between the initial splitter and the final mixer do not exist because it would lead to a loss of freshwater.

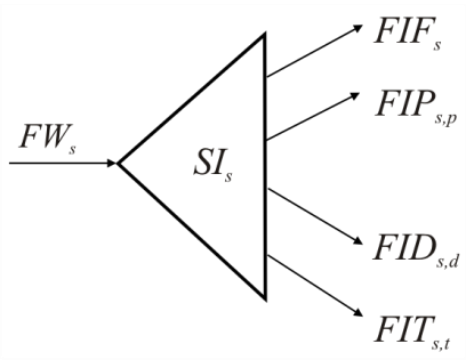

Figure 3. Initial splitter.

The overall material balance for the initial splitter is given by Eq. (1):

$$
F W_{s}=F I F_{s}+\sum_{p \in P U} F I P_{s, p}+\sum_{d \in D U} F I D_{s, d}+\sum_{t \in T U} F I T_{s, t} \quad \forall s \in S W
$$

For each of the corresponding flowrates, upper and lower bound constraints are formulated in terms of the binary variables that denote the existence of the pipes or these streams. 
$F I F_{s}^{L} \cdot y_{F I F_{s}} \leq F I F_{s} \leq F I F_{s}^{U} \cdot y_{F I F_{s}} \quad \forall s \in S W$

$F I P_{s, p}^{L} \cdot y_{F I P_{s, p}} \leq F I P_{s, p} \leq F I P_{s, p}^{U} \cdot y_{F I P_{s, p}} \quad \forall s \in S W, \forall p \in P U$

$F I D_{s, d}^{L} \cdot y_{F I D_{s, d}} \leq F I D_{s, d} \leq F I D_{s, d}^{U} \cdot y_{F I D_{s, d}} \quad \forall s \in S W, \forall d \in D U$

$F I T_{s, t}^{L} \cdot y_{F I T_{s, t}} \leq F I T_{s, t} \leq F I T_{s, t}^{U} \cdot y_{F I T_{s, t}} \quad \forall s \in S W, \forall t \in T U$

\subsection{Mixer process units}

The mixer process unit $M P U_{p}$, shown in Figure 4, consists of a set of inlet streams from the splitter source unit, the splitter treatment unit, the initial splitter, and the splitter process unit. An outlet stream from the mixer process unit is directed to the process unit.

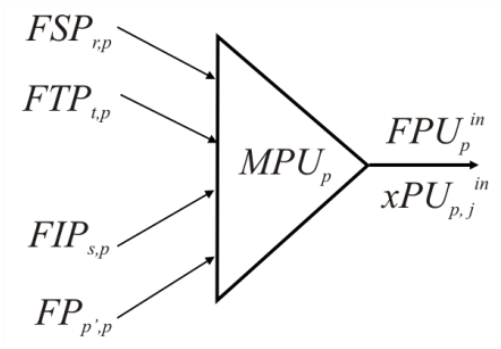

Figure 4. Mixer process unit.

The overall material balance for the mixer process unit is given by Eq. (6) and the mass balance for each contaminant $j$ by Eq. (7), which involves bilinear terms:

$$
\begin{aligned}
& F P U_{p}^{i n}=\sum_{r \in S U} F S P_{r, p}+\sum_{t \in T U} F T P_{t, p}+\sum_{s \in S W} F I P_{s, p}+\sum_{\substack{p^{\prime} \in P U \\
p \neq p^{\prime}, R_{p}=0}} F P_{p^{\prime}, p}+\sum_{\substack{p^{\prime} \in P U \\
R_{p}=1}} F P_{p^{\prime}, p}, \quad \forall p \in P U \\
& F P U_{p}^{i n} \cdot x P U_{p, j}^{i n}=\sum_{r \in S U} F S P_{r, p} \cdot x S U_{r, j}^{\text {out }}+\sum_{t \in T U} F T P_{t, p} \cdot x S T U_{t, j}^{\text {out }}+\sum_{s \in S W} F I P \cdot x W_{s, j}^{\text {in }} \\
& +\sum_{\substack{p^{\prime} \in P U \\
p \neq p^{\prime}, R_{p}=0}} F P_{p^{\prime}, p} \cdot x S P U_{p^{\prime}, j}^{\text {out }}+\sum_{\substack{p^{\prime} \in P U \\
R_{p}=1}} F P_{p^{\prime}, p} \cdot x S P U_{p^{\prime}, j}^{\text {out }}, \quad \forall p \in P U, \forall j
\end{aligned}
$$

\subsection{Process units}

The process unit $P U_{p}$, shown in Figure 5, consists of an inlet stream $F P U_{p}^{i n}$ from the mixer process unit and an outlet stream $F P U_{p}^{\text {out }}$ from the process unit. In the mass transfer process unit there is direct contact, usually countercurrent, between a contaminant-rich process stream and a contaminantlean water stream. These units can be represented as a "true" contaminant-rich process streams. In 
this case during the mass-transfer process the contaminants $L P U_{p, j}$ (pollutants) are transferred from the process streams to the water. The contaminant concentration in the process stream is reduced, while the contaminant concentration increases in the water stream (Mann and Liu 1999).

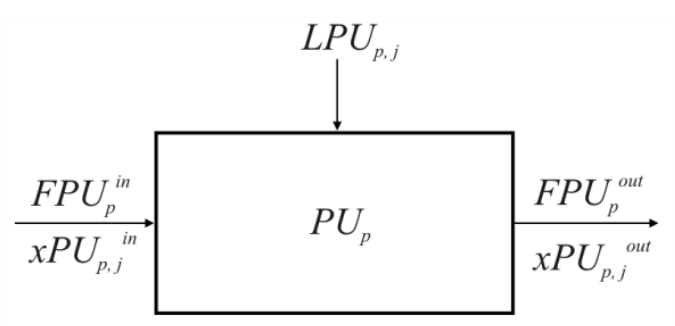

Figure 5. Process unit.

The inlet and outlet water flows for the process unit are equal. The overall material balance is given by Eq. (8) and the mass balance equation for each contaminant $j$ by Eq. (9):

$F P U_{p}^{\text {in }}=F P U_{p}^{\text {out }} \quad \forall p \in P U$

$F P U_{p}^{\text {in }} \cdot x P U_{p, j}^{\text {in }}+L P U_{p, j} \cdot 10^{3}=F P U_{p}^{\text {out }} \cdot x P U_{p, j}^{\text {out }} \quad \forall p \in P U, \forall j$

The outlet concentrations in the flows from the process units and the inlet concentrations to the splitter process units are the same as shown in Eq. (10):

$x P U_{p, j}^{\text {out }}=x S P U_{p, j}^{\text {in }} \quad \forall p \in P U, \forall j$

\subsection{Splitter process units}

The splitter process unit $S P U_{p}$ shown in Figure 6 consists of an inlet stream from the process unit, and a set of outlet streams directed to the final mixer, the mixer treatment unit, the mixer demand unit, and the mixer process unit. The overall material balance for the splitter process unit is given by Eq. (11):

$$
F P U_{p}^{\text {out }}=F P O_{p}+\sum_{t \in T U} F P T_{p, t}+\sum_{d \in D U} F P D_{p, d}+\sum_{\substack{p^{\prime} \in P U \\ p \neq p^{\prime}, R_{p}=0}} F P_{p^{\prime}, p}+\sum_{\substack{p^{\prime} \in P U \\ R_{p}=1}} F P_{p^{\prime}, p} \quad \forall p \in P U
$$

Figure 6. Splitter process unit. 
The contaminant concentration of every stream leaving the splitter is equal to the contaminant concentration of the inlet stream and this equality is given by Eq. (12):

$$
x S P U_{p, j}^{\text {out }}=x S P U_{p, j}^{\text {in }} \quad \forall p \in P U, \forall j
$$

The lower and upper bound constraints that relate the 0-1 variables with the flows between the splitter process units and all mixers in the network are given as follows.

$$
\begin{array}{lc}
F P O_{p}^{L} \cdot y_{F P O_{p}} \leq F P O_{p} \leq F P O_{p}^{U} \cdot y_{F P O_{p}} & \forall p \in P U \\
F P T_{p, t}^{L} \cdot y_{F P T_{p, t}} \leq F P T_{p, t} \leq F P T_{p, t}^{U} \cdot y_{F P T_{p, t}} & \forall p \in P U, \forall t \in T U \\
F P D_{p, d}^{L} \cdot y_{F P D_{p, d}} \leq F P D_{p, d} \leq F P D_{p, d}^{U} \cdot y_{F P D_{p, d}} & \forall p \in P U, \forall d \in D U \\
F P_{p^{\prime}, p}^{L} \cdot y_{F P_{p^{\prime}, p}} \leq F P_{p^{\prime}, p} \leq F P_{p^{\prime}, p}^{U} \cdot y_{F P_{p^{\prime}, p}} & \forall p \in P U, \forall p^{\prime} \in P U, p^{\prime} \neq p, R_{p}=0 \\
F P_{p^{\prime}, p}^{L} \cdot y_{F P_{p^{\prime}, p}} \leq F P_{p^{\prime}, p} \leq F P_{p^{\prime}, p}^{U} \cdot y_{F P_{p^{\prime}, p}} & \forall p \in P U, \forall p^{\prime} \in P U, p^{\prime} \neq p, R_{p}=1
\end{array}
$$

\subsection{Demand and source process units}

In many processes there is loss of water that cannot be re-used in a water-using operation. This unit represents a water demand unit or water sink. Cooling towers are typical process units where water is lost by evaporation. The demand process unit is shown in Figure 7.

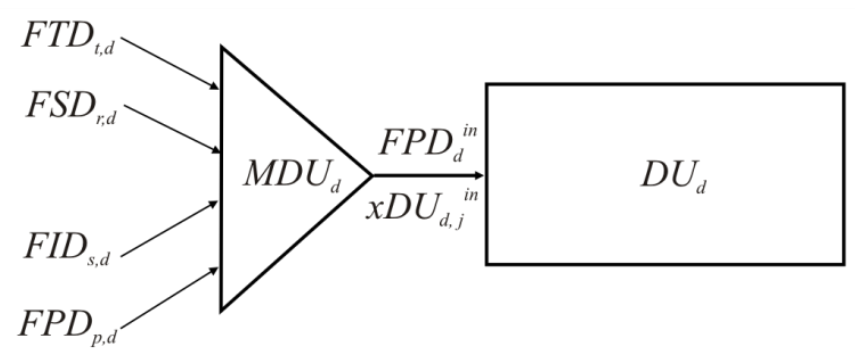

Figure 7. Demand process unit.

The mixer demand unit $M D U_{d}$ unit shown in Figure 7 consists of a set of inlet streams from the splitter treatment unit, the splitter source unit, initial splitter, and the splitter process unit. An outlet stream from the mixer demand unit is directed to the demand unit. The overall material balance for the mixer demand unit is given by Eq. (18) and the mass balance for each contaminant $j$ by Eq. (19):

$$
F D U_{d}^{i n}=\sum_{t \in T U} F T D_{t, d}+\sum_{r \in S U} F S D_{r, d}+\sum_{s \in S W} F I D_{s, d}+\sum_{p \in P U} F P D_{p, d} \quad \forall d \in D U
$$




$$
\begin{aligned}
F D U_{d}^{\text {in }} \cdot x S D U_{d, j}^{\text {in }} & =\sum_{t \in T U} F T D_{t, d} \cdot x S T U_{t, j}^{\text {out }}+\sum_{r \in S U} F S D_{r, d} \cdot x S U_{r, j}^{\text {out }}+\sum_{s \in S W} F I D_{s, d} \cdot x W_{s, j}^{\text {in }}+ \\
& +\sum_{p \in P U} F P D_{p, d} \cdot x S P U_{p, j}^{\text {out }} \quad \forall d \in D U, \forall j
\end{aligned}
$$

Additionally, the contaminant concentration at the demand inlet has to be less or equal to the maximum allowed contaminant concentration:

$$
x S D U_{d, j}^{i n} \leq x S D U_{d, j}^{i n, \max } \quad \forall d \in D U, \forall j
$$

From some water-using operations water is available for re-use in other operations. These units represent water sources or gains of water. The splitter source unit $S S U_{r}$ unit shown in Figure 8 consists of an inlet stream from the source unit and a set of outlet streams directed to the mixer process unit, the final mixer, the mixer treatment unit, and the mixer demand unit.

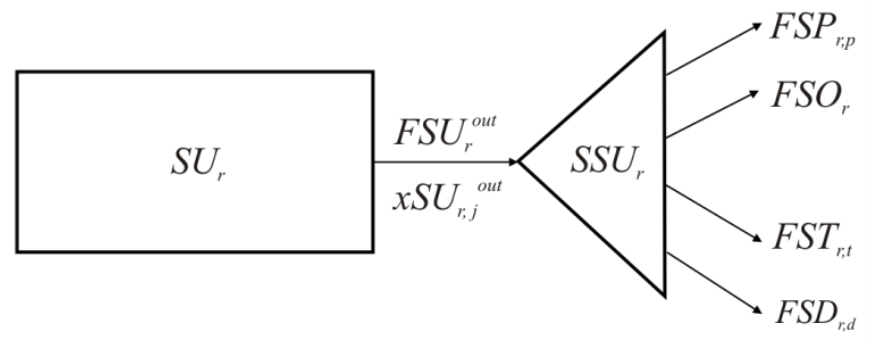

Figure 8. Source process unit.

The overall material balance for the splitter source unit is given by Eq. (21). Notice, that the contaminant concentration at the outlet of the source unit and the outlet of the splitter source unit is the same.

$$
F S U_{r}^{\text {out }}=\sum_{p \in P U} F S P_{r, p}+F S O_{r}+\sum_{t \in T U} F S T_{r, t}+\sum_{d \in D U} F S D_{r, d} \quad \forall r \in S U
$$

The lower and upper bound constraints that relate the flows and 0-1 variables for the streams between the splitter source units and all mixers in the network are given as follows.

$$
\begin{array}{lc}
F S P_{r, p}^{L} \cdot y_{F S D_{r, p}} \leq F S P_{r, p} \leq F S P_{r, p}^{U} \cdot y_{F S P_{r, p}} & \forall r \in S U, \forall p \in P U \\
F S O_{r}^{L} \cdot y_{F S O_{r}} \leq F S O_{r} \leq F S O_{r}^{U} \cdot y_{F S O_{r}} & \forall r \in S U \\
F S T_{r, t}^{L} \cdot y_{F S T_{r, t}} \leq F S T_{r, t} \leq F S T_{r, t}^{U} \cdot y_{F S T_{r, t}} & \forall r \in S U, \forall t \in T U \\
F S D_{r, d}^{L} \cdot y_{F S D_{r, d}} \leq F S D_{r, d} \leq F S D_{r, d}^{U} \cdot y_{F S D_{r, d}} & \forall r \in S U, \forall d \in D U
\end{array}
$$




\subsection{Mixer treatment units}

The mixer treatment unit $M T U_{t}$ shown in Figure 9 consists of a set of inlet streams from the splitter source unit, the initial splitter, the splitter process unit, and the splitter treatment unit.

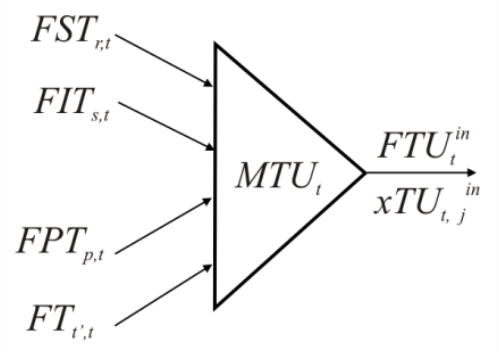

Figure 9. Mixer treatment unit.

The overall material balance for the mixer that is placed in front of the treatment unit is given by Eq. (26) and the mass balance for each contaminant $j$ by Eq. (27):

$$
\begin{gathered}
F T U_{t}^{\text {in }}=\sum_{r \in S U} F S T_{r, t}+\sum_{s \in S W} F I T_{s, t}+\sum_{p \in P U} F P T_{p, t}+\sum_{\substack{t^{\prime} \in T U \\
t \in t^{\prime}, R_{t}=0}} F T_{t^{\prime}, t}+\sum_{\substack{t^{\prime} \in T U \\
R_{t}=1}} F T_{t^{\prime}, t} \quad \forall t \in T U \\
F T U_{t}^{\text {in }} \cdot x T U_{t, j}^{\text {in }}=\sum_{r \in S U} F S T_{r, t} \cdot x S U_{r, j}^{\text {out }}+\sum_{s \in S W} F I T_{s, t} \cdot x W_{s, j}^{W}+\sum_{p \in P U} F P T_{p, t} \cdot x S P U_{p, j}^{\text {out }} \\
+\sum_{\substack{t^{\prime} \in T U \\
t \neq t^{\prime}, R_{T U}=0}} F T_{t^{\prime}, t} \cdot x S T U_{t, j}^{\text {out }}+\sum_{\substack{t^{\prime} \in T U \\
R_{T U}=1}} F T_{t^{\prime}, t} \cdot x S T U_{t, j}^{\text {out }} \quad \forall t \in T U, \forall j
\end{gathered}
$$

\subsection{Treatment units}

The treatment unit $T U_{t}$, shown in Figure 10, consists of an inlet stream from the mixer treatment unit, and an outlet stream from the treatment unit. The inlet and outlet flows for a treatment unit are equal and the overall material balance is given by Eq. (28).

$F T U_{t}^{\text {in }}=F T U_{t}^{\text {out }} \quad \forall t \in T U$

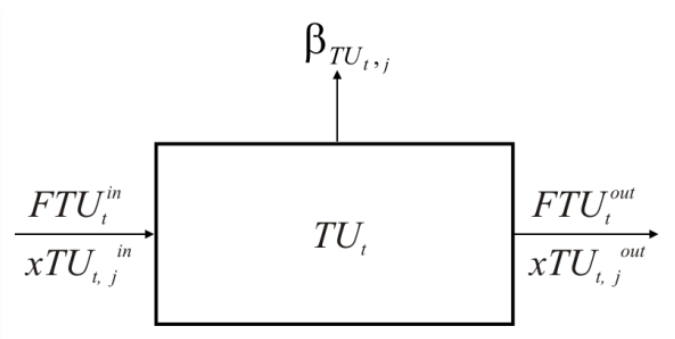

Figure 10. Treatment unit. 
The mass balance equation for each contaminant $j$ for the treatment unit $t \in T U$ is assumed to be a linear function given by Eq. (29).

$x T U_{t, j}^{\text {out }}=\beta_{T U t, j} \cdot x T U_{t, j}^{\text {in }} \quad \forall t \in T U, \forall j$

where $\beta_{T U t, j}=\left(1-R R_{t, j} / 100\right)$ for $\forall t \in T U, \forall j$.

\subsection{Splitter treatment units}

The splitter treatment unit $S T U_{t}$ shown in Figure 11 consists of an inlet stream from the treatment unit and a set of outlet streams directed to the mixer demand unit, the mixer process unit, the final mixer and the mixer treatment unit.

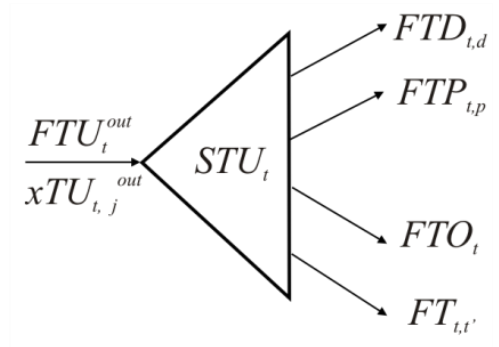

Figure 11. Splitter treatment unit.

The overall material balance for the splitter process unit is given by equation Eq. (30) and the equality of concentrations at the treatment unit and the splitter treatment unit by Eq. (31):

$$
\begin{aligned}
& F T U_{t}^{\text {out }}=\sum_{d \in D U} F T D_{t, d}+\sum_{p \in P U} F T P_{t, p}+F T O_{t}+\sum_{\substack{t^{\prime} \in T U \\
t \neq t^{\prime}, R_{t}=0}} F T_{t^{\prime}, t}+\sum_{\substack{t^{\prime} \in T U \\
R_{t}=1}} F T_{t^{\prime}, t} \quad \forall t \in T U \\
& x S T U_{t, j}^{\text {out }}=x T U_{t, j}^{\text {out }} \quad \forall t \in T U, \forall j
\end{aligned}
$$

The lower and upper bound constraints that relate the flows and 0-1 variables for streams between the splitter treatment units and all mixers in the network are given as follows.

$$
\begin{aligned}
& F T D_{t, d}^{L} \cdot y_{F T D_{t, d}} \leq F T D_{t, d} \leq F T D_{t, d}^{U} \cdot y_{F T D_{t, d}} \quad \forall t \in T U, \forall d \in D U \\
& F T P_{t, p}^{L} \cdot y_{F T P_{t, p}} \leq F T P_{t, p} \leq F T P_{t, p}^{U} \cdot y_{F T P_{t, p}} \quad \forall t \in T U, \forall p \in P U \\
& F T O_{t}^{L} \cdot y_{F T O_{t}} \leq F T O_{t} \leq F T O_{t}^{U} \cdot y_{F T O_{t}} \quad \forall t \in T U \\
& F T_{t^{\prime}, t}^{L} \cdot y_{F T_{t^{\prime}, t}} \leq F T_{t^{\prime}, t} \leq F T_{t^{\prime}, t}^{U} \cdot y_{F T_{t^{\prime}, t}} \quad \forall t \in T U, \forall t^{\prime} \in T U, t^{\prime} \neq t, R_{t}=0 \\
& F T_{t^{\prime}, t}^{L} \cdot y_{F T_{t^{\prime}, t}} \leq F T_{t^{\prime}, t} \leq F T_{t^{\prime}, t}^{U} \cdot y_{F T_{t^{\prime}, t}} \quad \forall t \in T U, \forall t^{\prime} \in T U, R_{t}=1
\end{aligned}
$$




\subsection{Final mixer}

The final mixer unit $M F$ shown in Figure 12 consists of a set of inlet streams from the initial splitter, the splitter process unit, the splitter treatment unit, and the splitter source unit.

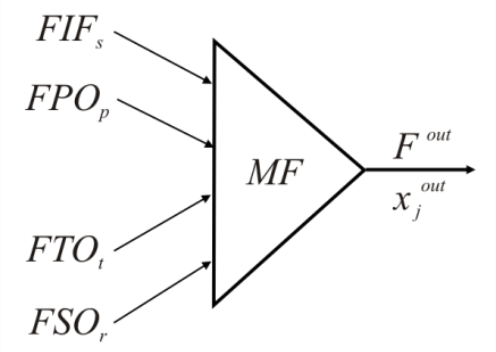

Figure 12. Final mixer.

The overall material balance for the final mixer is given by Eq. (37) and the mass balance equation for each contaminant $j$ by Eq. (38):

$$
\begin{aligned}
F^{\text {out }}= & \sum_{s \in S W} F I F_{s}+\sum_{p \in P U} F P O_{p}+\sum_{t \in T U} F T O_{t}+\sum_{r \in S U} F S O_{r} \\
F^{\text {out }} \cdot x_{j}^{\text {out }}= & \sum_{s \in S W} F I F_{s} \cdot x W_{s, j}^{\text {in }}+\sum_{p \in P U} F P O_{p} \cdot x S P U_{p, j}^{\text {out }}+\sum_{t \in T U} F T O_{t} \cdot x S T U_{t, j}^{\text {out }} \\
& +\sum_{r \in S U} F S O_{r} \cdot x S U_{r, j}^{\text {out }} \quad \forall j
\end{aligned}
$$

Using the binary variables for the existence of pipe connections, the specification for a maximum number of these connections in the network is given by Eq. (39). Using this constraint it is possible to control the complexity of the piping network.

$$
\begin{aligned}
& \sum_{s \in S W} \sum_{p \in P U} y_{F I P_{s, p}}+\sum_{s \in S W} \sum_{t \in T U} y_{F I T_{s, t}}+\sum_{s \in S W} y_{F I F_{s}}+\sum_{\substack{p^{\prime} \in P U \\
p^{\prime} \neq p, R_{p}=0}} \sum_{p \in P U} y_{F P_{p^{\prime}, p}}+\sum_{\substack{p^{\prime} \in P U \\
R_{P U}=1}} \sum_{p \in P U} y_{F P_{p^{\prime}, p}}+\sum_{p \in P U} y_{F P O_{p}} \\
& +\sum_{p \in P U} \sum_{t \in T U} y_{F P T_{p, t}}+\sum_{\substack{t^{\prime} \in T U \\
t^{\prime \neq t}, R_{t}=0}} \sum_{t \in T U} y_{F T_{t^{\prime}, t}}+\sum_{\substack{t^{\prime} \in T U \\
R_{t}=1}} \sum_{t \in T U} y_{F T_{t^{\prime}, t}}+\sum_{t \in T U} y_{F T O_{t}}+\sum_{t \in T U} \sum_{p \in P U} y_{F T P_{t, p}} \\
& +\sum_{s \in S W} \sum_{d \in D U} y_{F I D_{s, d}}+\sum_{p \in P U} \sum_{d \in D U} y_{F P D_{p, d}}+\sum_{t \in T U} \sum_{d \in D U} y_{F T D_{t, d}}+\sum_{r \in S U} \sum_{d \in D U} y_{F S D_{r, d}}+\sum_{r \in S U} y_{F S O_{r}} \\
& +\sum_{r \in S U} \sum_{t \in T U} y_{F S T_{r, t}}+\sum_{r \in S U} \sum_{p \in P U} y_{F S P_{r, p}} \leq N_{\max }
\end{aligned}
$$

\subsection{Objective function of the NLP and MINLP model}

When binary variables are excluded with their corresponding lower and upper bound constraints, the MINLP model in the previous section reduces to an NLP. The objective function of the NLP water network problems can simply be formulated to minimize the total consumption of freshwater 
for network plant operation and the total amount of wastewater treated in treatment operations for an integrated system (Karuppiah \& Grossmann, 2006).

$\min Z=\sum_{s \in S W} F W_{s}+\sum_{t \in T U} F T U_{t}^{\text {out }}$

For separate subsystems consisting of water-using or wastewater treatment operations the objective function is to either minimize the total consumption of freshwater or the total amount of wastewater treated in treatment operations, respectively.

$$
\begin{aligned}
& \min Z=\sum_{s \in S W} F W_{s} \\
& \min Z=\sum_{t \in T U} F T U_{t}^{\text {out }}
\end{aligned}
$$

A more accurate objective function is to minimize the total network cost consisting of the cost of freshwater, the cost of investment on treatment units and the operating cost for the treatment units. This type of objective function is used in many papers to optimize the water network problems.

$$
\min Z=H \cdot \sum_{s \in S W} F W_{s} \cdot C F W_{s}+A R \cdot \sum_{t \in T U} I C_{t} \cdot\left(F T U_{t}^{\text {out }}\right)^{\alpha}+H \cdot \sum_{t \in T U} O C_{t} \cdot F T U_{t}^{\text {out }}
$$

Furthermore, in the case of separate subsystems consisting of water-using or wastewater treatment operations the objective function is to minimize the total cost of water, or the total cost of investment on treatment units and operating cost for the treatment units:

$$
\begin{aligned}
& \min Z=H \cdot \sum_{s \in S W} F W_{s} \cdot C F W_{s} \\
& \min Z=A R \cdot \sum_{t \in T U} I C_{t} \cdot\left(F T U_{t}^{\text {out }}\right)^{\alpha}+H \cdot \sum_{t \in T U} O C_{t} \cdot F T U_{t}^{\text {out }}
\end{aligned}
$$

In most papers, the cost of the network piping and the cost of water pumping through pipes are not considered. Here, we introduce these costs in the objective function when the water network problem is formulated as an MINLP problem. The objective function is to minimize the total network cost given by Eq. (46):

$$
\min Z=C_{\text {water }}+I C_{\text {treatment }}+O C_{\text {treatment }}+O C_{\text {waterpumping }}+I C_{\text {pipes }}
$$

Here $C_{\text {water }}$ is the yearly cost of water for the network plant operation; $I C_{\text {treatment }}$ is the investment cost for treatment units; $O C_{\text {treatment }}$ is the yearly operating cost for treatment units; $O C_{\text {pumping }}$ is the yearly operating cost for pumping water through pipes in the network; $I C_{\text {pipes }}$ is the investment cost of the pipes in the network. The annualized costs are expressed for each term in Eq. (46) as follows.

$$
C_{\text {water }}=H \cdot \sum_{s \in S W} F W_{s} \cdot C F W_{s}
$$




$$
\begin{aligned}
& I C_{\text {treatment }}=A R \cdot \sum_{t \in T U} I C_{t} \cdot\left(F T U_{t}^{\text {out }}\right)^{\alpha} \\
& O C_{\text {treatment }}=H \cdot \sum_{t \in T U} O C_{t} \cdot F T U_{t}^{o u t} \\
& O C_{\text {pumping }}=H \cdot\left[\sum_{s \in S W} \sum_{p \in P U} F I P_{s, p} \cdot P M_{p}+\sum_{s \in S W} \sum_{t \in T U} F I T_{s, t} \cdot P M_{t}+\sum_{t \in T U} \sum_{p \in P U} F T P_{t, p} \cdot P M_{t}\right. \\
& +\sum_{\substack{p^{\prime} \in P U \\
R_{p}=1}} \sum_{p \in P U} F P_{p^{\prime}, p} \cdot P M_{p^{\prime}}+\sum_{\substack{p^{\prime} \in P U \\
p^{\prime} \neq p, R_{p}=0}} \sum_{p \in P U} F P_{p^{\prime}, p} \cdot P M_{p^{\prime}}+\sum_{p \in P U} \sum_{t \in T U} F P T_{p, t} \cdot P M_{p} \\
& +\sum_{p \in P U} F P O_{p} \cdot P M_{p}+\sum_{t \in T U} F T O_{t} \cdot P M_{t}+\sum_{\substack{t^{\prime} \in T U \\
R_{t}=1}} \sum_{t \in T U} F T_{t^{\prime}, t} \cdot P M_{t^{\prime}} \\
& +\sum_{\substack{t^{\prime} \in T U \\
t^{\prime} \neq t, R_{T U}=0}} \sum_{t \in T U} F T_{t^{\prime}, t} \cdot P M_{t^{\prime}}+\sum_{s \in S W} \sum_{d \in D U} F I D_{s, d} \cdot P M_{d}+\sum_{p \in P U} \sum_{d \in D U} F P D_{p, d} \cdot P M_{d} \\
& +\sum_{t \in T U} \sum_{d \in D U} F T D_{t, d} \cdot P M_{d}+\sum_{r \in S U} \sum_{d \in D U} F S D_{r, d} \cdot P M_{d}+\sum_{r \in S U} \sum_{p \in P U} F S P_{r, p} \cdot P M_{p} \\
& \left.+\sum_{r \in S U} \sum_{t \in T U} F S T_{r, t} \cdot P M_{t}+\sum_{r \in S U} F S O_{r} \cdot P M_{r}\right] \\
& I C_{\text {pipes }}=A R \cdot\left[\sum_{t \in T U} \sum_{p \in P U}\left(C P_{t} \cdot y_{F T P_{t, p}}+I P_{t} \cdot\left(F T P_{t, p}\right)\right)^{\gamma}+\sum_{\substack{p^{\prime} \in P U \\
R_{p}=1}} \sum_{p \in P U}\left(C P_{p^{\prime}} \cdot y_{F P_{p^{\prime}, p}}+I P_{p^{\prime}} \cdot\left(F P_{p^{\prime}, p}\right)^{\gamma}\right)\right. \\
& +\sum_{\substack{p^{\prime} \in P U \\
p^{\prime} \neq p, R_{p}=0}} \sum_{p \in P U}\left(C P_{p^{\prime}} \cdot y_{F P_{p^{\prime}, p}}+I P_{p^{\prime}} \cdot\left(F P_{p^{\prime}, p}\right)^{\gamma}\right)+\sum_{p \in P U} \sum_{t \in T U}\left(C P_{p} \cdot y_{F P T_{p, t}}+I P_{p} \cdot\left(F P T_{p, t}\right)^{\gamma}\right) \\
& +\sum_{p \in P U}\left(C P_{p} \cdot y_{F P O_{p}}+I P_{p} \cdot\left(F P O_{p}\right)^{\gamma}\right)+\sum_{t \in T U}\left(C P_{t} \cdot y_{F T O_{t}}+I P_{t} \cdot\left(F T O_{t}\right)^{\gamma}\right) \\
& +\sum_{\substack{t^{\prime} \in T U \\
R_{t}=1}} \sum_{t \in T U}\left(C P_{t^{\prime}} \cdot y_{F T_{t^{\prime}, t}}+I P_{t^{\prime}} \cdot\left(F T_{t^{\prime}, t}\right)^{\gamma}\right)+\sum_{\substack{t^{\prime} \in T U \\
t^{\prime} \neq t, R_{T U}=0}} \sum_{t \in T U}\left(C P_{t^{\prime}} \cdot y_{F T_{t^{\prime}, t}}+I P_{t^{\prime}} \cdot\left(F T_{t^{\prime}, t}\right)^{\gamma}\right) \\
& +\sum_{s \in S W} \sum_{p \in P U}\left(C P_{p} \cdot y_{F I P_{s, p}}+I P_{p} \cdot\left(F I P_{s, p}\right)^{\gamma}\right)+\sum_{s \in S W} \sum_{t \in T U}\left(C P_{t} \cdot y_{F I T_{s, t}}+I P_{t} \cdot\left(F I T_{s, t}\right)^{\gamma}\right) \\
& +\sum_{s \in S W} \sum_{d \in D U}\left(C P_{d} \cdot y_{F I D_{s, d}}+I P_{d} \cdot\left(F I D_{s, d}\right)^{\gamma}\right)+\sum_{p \in P U} \sum_{d \in D U}\left(C P_{d} \cdot y_{F P D_{p, d}}+I P_{d} \cdot\left(F P_{s, d}\right)^{\gamma}\right) \\
& +\sum_{t \in T U} \sum_{d \in D U}\left(C P_{d} \cdot y_{F T D_{t, d}}+I P_{d} \cdot\left(F T D_{t, d}\right)^{\gamma}\right)+\sum_{r \in S U} \sum_{d \in D U}\left(C P_{d} \cdot y_{F S D_{r, d}}+I P_{d} \cdot\left(F S D_{r, d}\right)^{\gamma}\right) \\
& +\sum_{r \in S U}\left(C P_{r} \cdot y_{F S O_{r}}+I P_{r} \cdot\left(F S O_{r}\right)^{\gamma}\right)+\sum_{r \in S U} \sum_{t \in T U}\left(C P_{t} \cdot y_{F S T_{r, t}}+I P_{t} \cdot\left(F S T_{r, t}\right)^{\gamma}\right) \\
& +\sum_{r \in S U} \sum_{p \in P U}\left(C P_{p} \cdot y_{F S P_{r, p}}+I P_{p} \cdot\left(F S P_{r, p}\right)^{\gamma}\right]
\end{aligned}
$$




\section{Solution strategy}

The proposed model presented in the previous section corresponds to a nonconvex NLP or nonconvex MINLP problem. This problem is modeled in GAMS (Brooke, Kendrick et al. 1988). In this paper, the BARON (Sahinidis, 1996) and LINDOGlobal solvers are used for solving all water network problems to global optimality.

To significantly improve the strength of the lower bound for the global optimum we incorporate the cut proposed by Karuppiah and Grossmann (2006). The bound strengthening in the nonlinear model corresponds to the contaminant flow balances for the overall water network system and is given by equation:

$$
\begin{aligned}
& \sum_{s \in S W} F W_{s} \cdot x W_{s, j}^{\text {in }}+\sum_{p \in P U} L P U_{p, j} \cdot 10^{3}+\sum_{r \in S U} F S U_{r}^{\text {out }} \cdot x S U_{r, j}^{\text {out }}=\sum_{t \in T U}\left(1-\beta_{T U t, j}\right) \cdot F T U_{t}^{\text {in }} \cdot x T U_{t, j}^{\text {in }} \\
& +F^{\text {out }} \cdot x_{j}^{\text {out }}+\sum_{d \in D U} F D U_{d}^{\text {in }} \cdot x D U_{d, j}^{\text {in }} \quad \forall j
\end{aligned}
$$

where bilinear terms are involved for the treatment units and final mixing points. It is also worth pointing out that when solving nonconvex water network problems by the previously mentioned global optimization solvers, it is important to specify good variable bounds for all flowrates and concentrations in the water network. The reason is that these bounds are used in the convex envelopes for under and overestimating the nonconvexities (eg. secant for concave function or McCormick envelopes for bilinear terms). In the proposed model the bounds on the variables are represented as general equations as shown in the Appendix. They are obtained by physical inspection of the superstructure and by using logic specifications. Using the proposed model with the cuts by Karuppiah and Grossmann (2006) and the bounds in the Appendix we can effectively solve the NLP water network problems for different levels of complexity with multiple sources of water, multiple contaminants and more process and treatment units (large-scale problems). Also, the MINLP water network problems with a modest number of process units, treatment units, and contaminants can be effectively solved using the proposed model. However, for large-scale MINLP problems the global optimization solvers cannot find the optimal solution in reasonable computational time.

To circumvent this problem, we propose a solution strategy that can be used for solving large-scale industrial water network problems. The basic idea is shown in Figure 13. When the objective is to minimize the total network cost given by Eq. (43) without specifying a maximum number of piping connections, we solve the NLP problem in which the 0-1 variables and the upper and lower bound constraints are excluded. Once we obtain the solution of the NLP, we fix all zero flowrates in the network and update the variable bounds before solution of reduced the MINLP. In the case when we 
specify a maximum number of pipe segments, we solve first the relaxed MINLP problem. The 0-1 variables of the streams in the network with zero value we fix at zero and then solve the reduced MINLP. With this solution method we can control the complexity of the water network. After solving the MINLP problem we can solve it again by restricting the number of piping connections. It that case, we assign to the model a new number of piping connections using the equation $N_{\text {streams }}^{M I N L P}=N_{\text {streams }}^{M I N L P}-N$. Here $N$ is the number of piping connections for simplifying the network. Both, the NLP and reduced MINLP models are solved by a global optimization solver. While the rigorous global optimum cannot be guaranteed with the two-stage solution strategy, in our experience the global optimum is still obtained in most cases

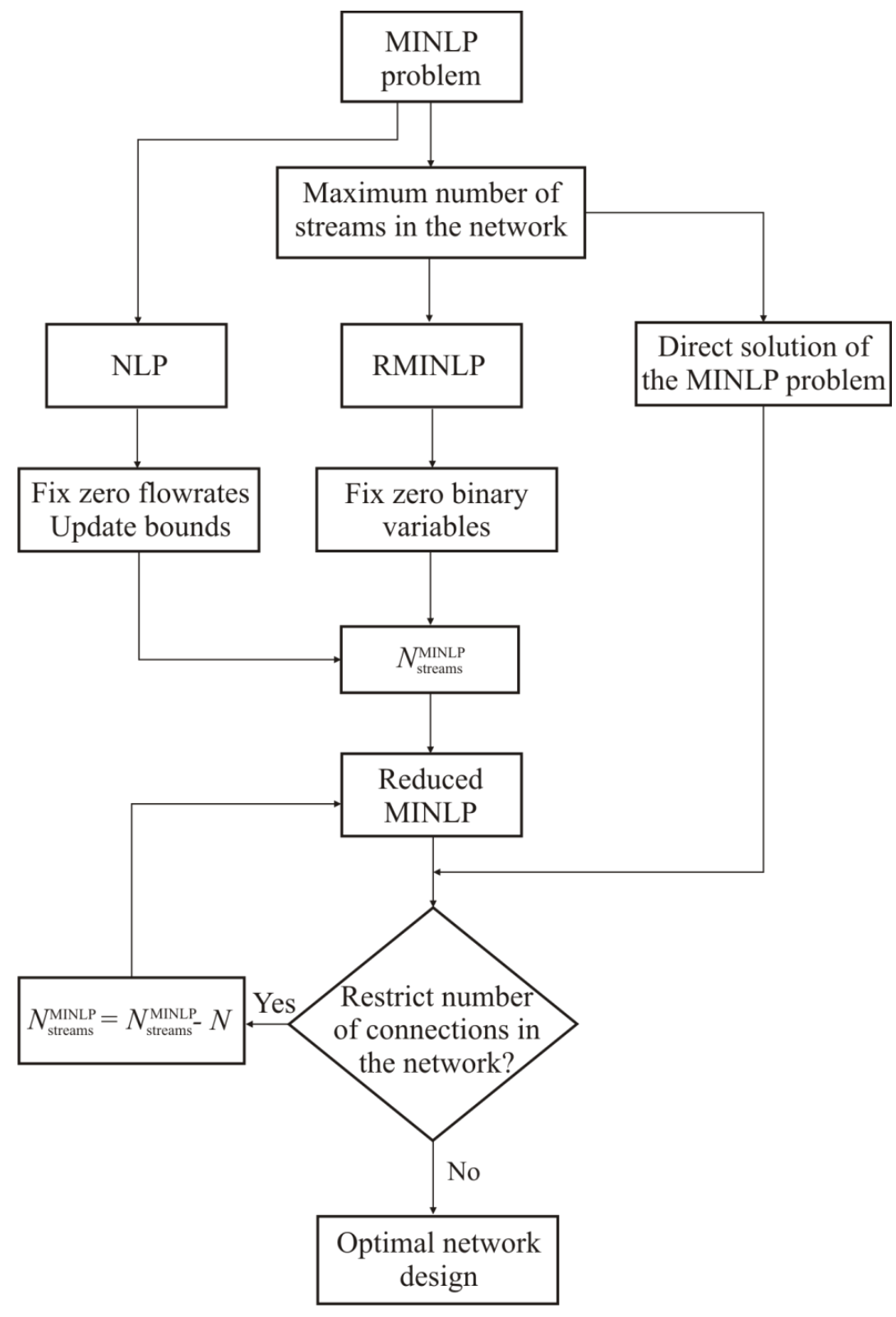

Figure 13. Two-stage solution strategy for solving the MINLP problems. 


\section{Examples}

In this section we present several examples to illustrate the proposed optimization models. All examples were implemented in GAMS 23.0 (Brooke, Kendrick, Meeraus, \& Raman, 1988) and solved on a HP Pavilion Notebook PC with 4 GB RAM memory, and Intel Core Duo $2 \mathrm{GHz}$ processor. The general purpose global optimization solver BARON (Sahinidis, 1996) or LINDOGlobal are used for solving the examples to global optimality. Model statistics, problems sizes and computational times are reported at the end of the six example problems.

\subsection{Example 1}

In this example we illustrate the advantage of using local recycles around the process units in the network. Moreover, it is shown how the complexity of the water networks can be controlled by restricting the number of piping connections. The water network superstructure for this example is shown in Figure 14. It consists of two process, two treatment units and a single source of water.

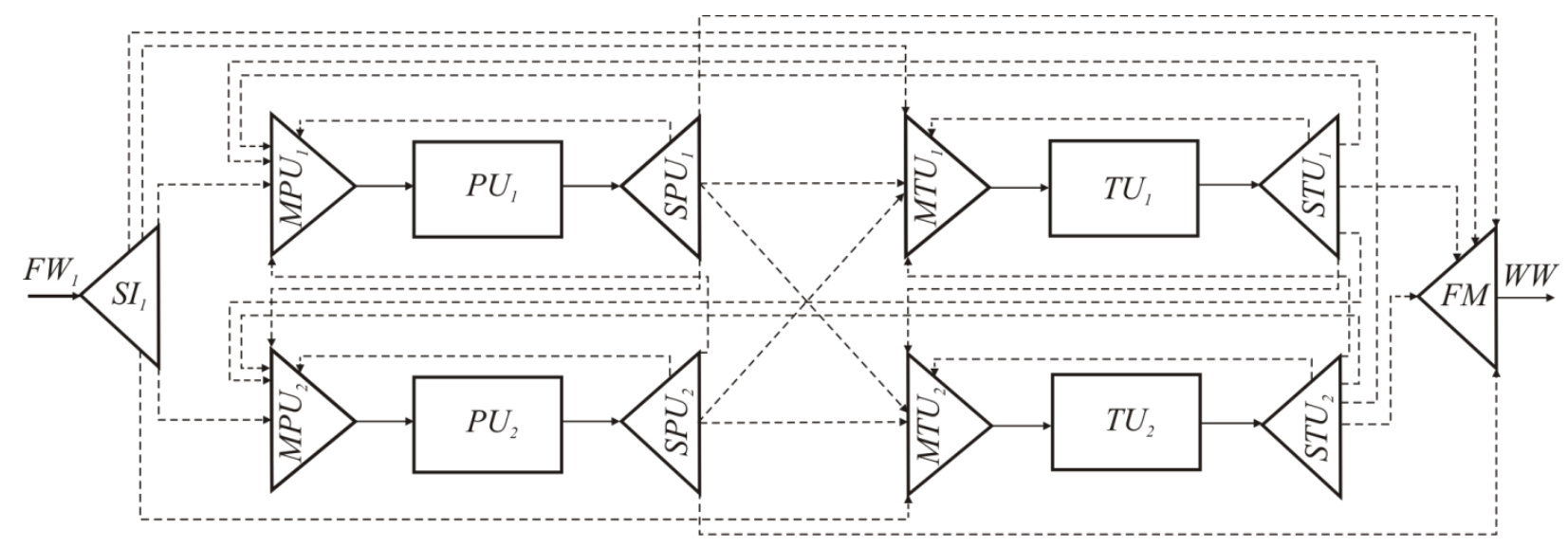

Figure 14. Water network superstructure for Example 1.

Data of process units, treatment units and contaminants are given in Tables 1 and 2, respectively and are taken from Karuppiah and Grossmann (2006).

Table 1. Data of process units.

\begin{tabular}{llllll}
\hline Unit & Flowrate $(\mathrm{t} / \mathrm{h})$ & \multicolumn{2}{l}{$\begin{array}{l}\text { Discharge load } \\
(\mathrm{kg} / \mathrm{h})\end{array}$} & \multicolumn{2}{l}{$\begin{array}{l}\text { Maximum inlet } \\
\text { concentration }(\mathrm{ppm})\end{array}$} \\
\cline { 3 - 6 } & $\mathrm{A}$ & $\mathrm{B}$ & $\mathrm{A}$ & $\mathrm{B}$ \\
\hline $\mathrm{PU}_{1}$ & 40 & 1 & 1.5 & 0 & 0 \\
$\mathrm{PU}_{2}$ & 50 & 1 & 1 & 50 & 50 \\
\hline
\end{tabular}


Table 2. Data of treatment units.

\begin{tabular}{lccccc}
\hline Unit & $\begin{array}{c}\text { \% removal for } \\
\text { contaminant }\end{array}$ & $\begin{array}{c}\text { IC (Investment } \\
\text { cost coefficient) }\end{array}$ & $\begin{array}{c}\text { OC (Operating } \\
\text { cost coefficient) }\end{array}$ & $\alpha$ \\
\cline { 2 - 6 } & $\mathrm{A}$ & $\mathrm{B}$ & & 1 & 0.7 \\
\hline $\mathrm{TU}_{1}$ & 95 & 0 & 16800 & 0.0067 & 0.7 \\
$\mathrm{TU}_{2}$ & 0 & 95 & 12600 & & \\
\hline
\end{tabular}

Each treatment unit can remove only one contaminant. The environmental discharge limit for contaminant $\mathrm{A}$ and contaminant $\mathrm{B}$ is $10 \mathrm{ppm}$. The freshwater cost is assumed to be $\$ 1 /$ ton, the annualized factor for investment on the treatment units is taken to be 0.1 , and the total time for the network plant operation in a year is assumed to be $8000 \mathrm{~h}$. We formulated the problem as the nonconvex Nonlinear Programming (NLP) where the objective function is to minimize the total network cost given by equation (43). The global optimization results are given in Table 3 .

Table 3. Optimization results for the NLP water network problem.

\begin{tabular}{|c|c|c|}
\hline & Without recycle & With recycle \\
\hline Freshwater cost & $\$ 320,000$ & $\$ 320,000$ \\
\hline Investment cost of treatment units & $\$ 37,440$ & $\$ 33,585.3$ \\
\hline Operating costs of treatment units & $\$ 238,723.6$ & $\$ 230,431.6$ \\
\hline Total cost & $\$ 596,163.6$ & $\$ 584,016.9$ \\
\hline
\end{tabular}

With the local recycle around the process unit it is possible to meet both flowrate and contaminant constraints at the process unit inlets and have lower total network cost (\$584,016.9/year) compared to the case without local recycle $(\$ 596,163.6 /$ year$)$.

In addition to this, we solved the same example for the case when the investment cost for piping and the operating cost for pumping water inside pipes are included in the objective function (see equation 46). In this example, the fixed cost pertaining to the pipes is assumed to be $\$ 6$, the variable cost for each individual pipe $\$ 100$, and operating cost coefficient for pumping water through pipes \$0.006/ton (Karuppiah and Grossmann 2008). The global optimization results are given in Table 4. 
Table 4. Optimization results for the MINLP water network problem.

\begin{tabular}{|c|c|c|}
\hline & Without recycle & With recycle \\
\hline Freshwater cost & $\$ 320,000$ & $\$ 320,000$ \\
\hline Investment cost on pipes & $\$ 540.69$ & $\$ 546.3$ \\
\hline Operating cost for pumping water & $\$ 10,056.26$ & $\$ 9,427.84$ \\
\hline Investment cost of treatment units & $\$ 37,440.01$ & $\$ 33,585.32$ \\
\hline Operating costs of treatment units & $\$ 238,723.59$ & $\$ 230,431.64$ \\
\hline Total cost & $\$ 606,760.55$ & $\$ 593,991.1$ \\
\hline
\end{tabular}

It should be noted, that both the NLP and MINLP problem with or without local recycles have the same optimal design of water network as shown in Figures 15 and 16.

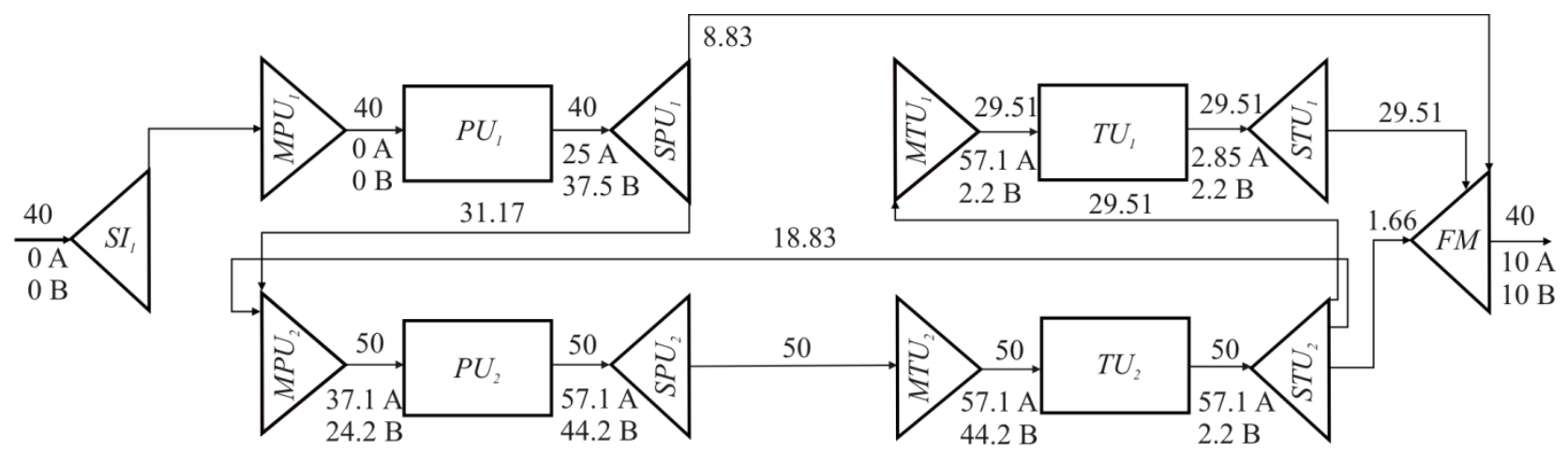

Figure 15. Optimal solution for the NLP and MINLP problem without local recycle.

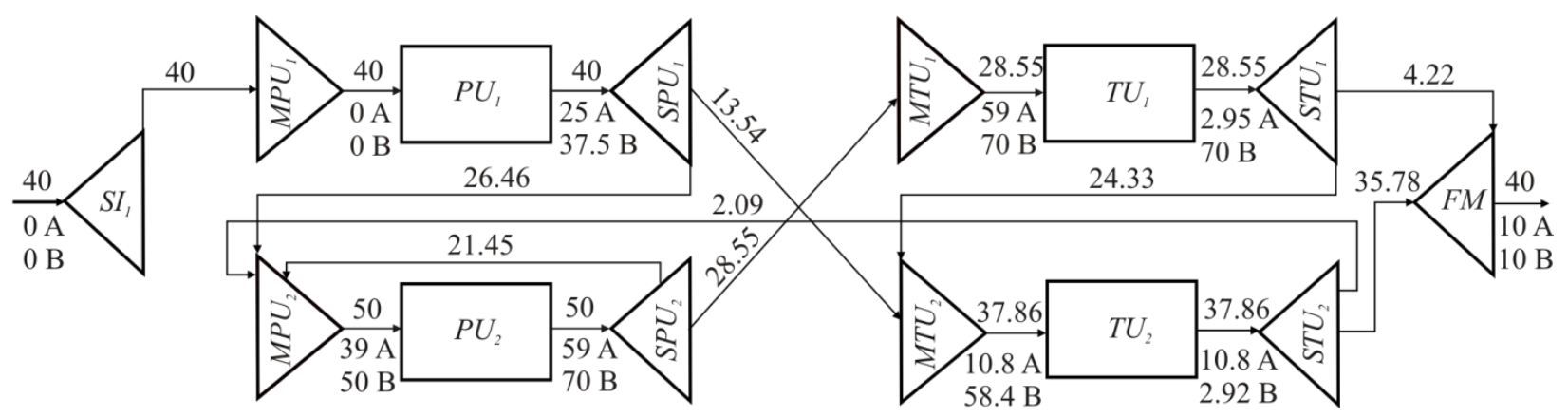

Figure 16. Optimal solution for the NLP and MINLP problem with local recycle.

As can be seen from Figures 15 and 16 the number of removable piping connections (streams between all splitters and mixers in the network) for the NLP and MINLP problem without local recycle is 8 and with local recycle it is 9. In addition, removable connections are shown in Figure 14 
as dashed lines that can be actually deleted from the superstructure. According to this, it is useful for the designer to have a tool which can be used to control network complexity. For the water networks given in Figures 15 and 16, and the same data for the MINLP problem, the results of the optimization by restricting the number of piping connections are shown in Table 5.

Table 5. Results of controlling the piping network complexity.

\begin{tabular}{|c|c|c|c|c|}
\hline \multirow{4}{*}{ Solver } & \multicolumn{2}{|c|}{ Total cost (\$/year) } & \multirow{2}{*}{\multicolumn{2}{|c|}{$\begin{array}{c}\text { Number of removable streams in the } \\
\text { network }\end{array}$}} \\
\hline & & & & \\
\hline & Without local & With local & Without local & With local \\
\hline & recycle & recycle & recycle & recycle \\
\hline \multirow{4}{*}{ BARON } & - & $593,991.11$ & - & 9 \\
\hline & $606,760.55$ & $596,012.94$ & 8 & 8 \\
\hline & $620,857.57$ & $613,610.77$ & 7 & 7 \\
\hline & $695,456.90$ & $691,610.36$ & 6 & 6 \\
\hline
\end{tabular}

The optimal network cost for the option without local recycle is $\$ 606,760.55 /$ year, and with local recycle $\$ 593,991.11 /$ year. In the first case the number of removable connections is 8 and in the second it is 9. In order to simplify the network we assigned to the design constraint Eq. (39) a new number of removable streams as shown in Figure 13. Figure 17 shows the results of the water network optimization for different number of removable streams.

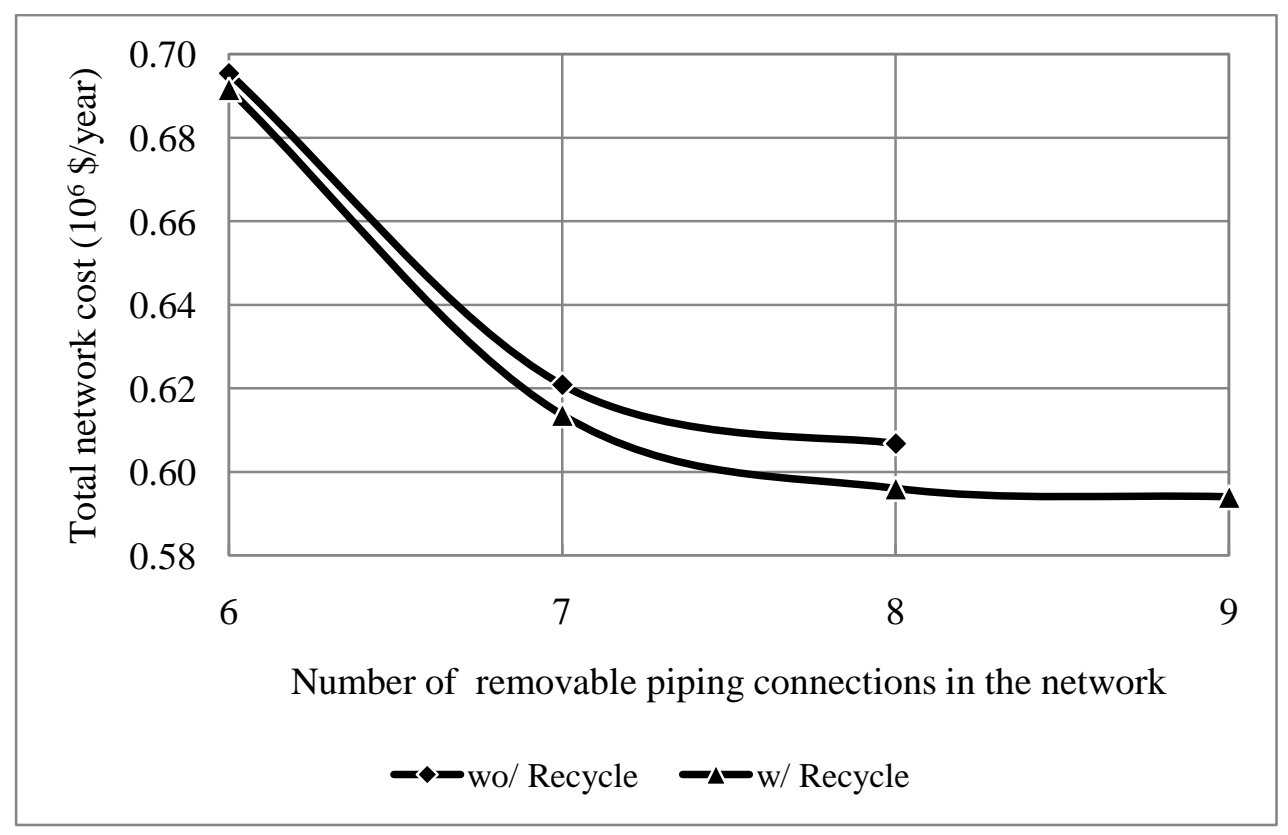

Figure 17. Controlling the network complexity by restricting number of removable connections. 
For instance, we can greatly simplify the water network with 6 removable piping connections as shown in Figures 18 and 19, while we still keep the freshwater consumption at $40 \mathrm{t} / \mathrm{h}$.

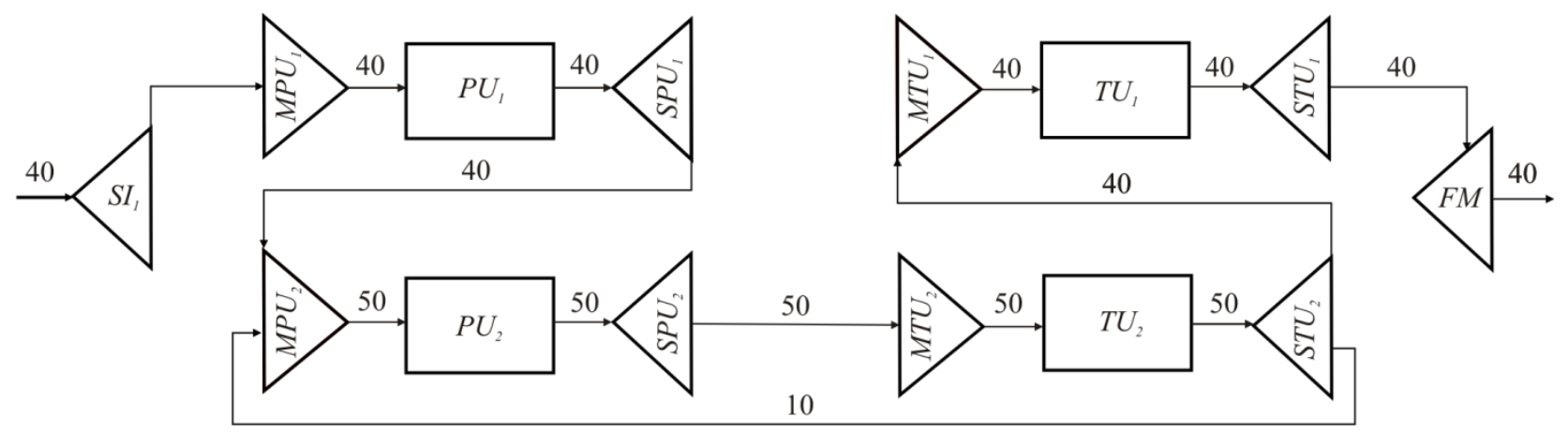

Figure 18. Water network with 6 removable streams and without local recycle.

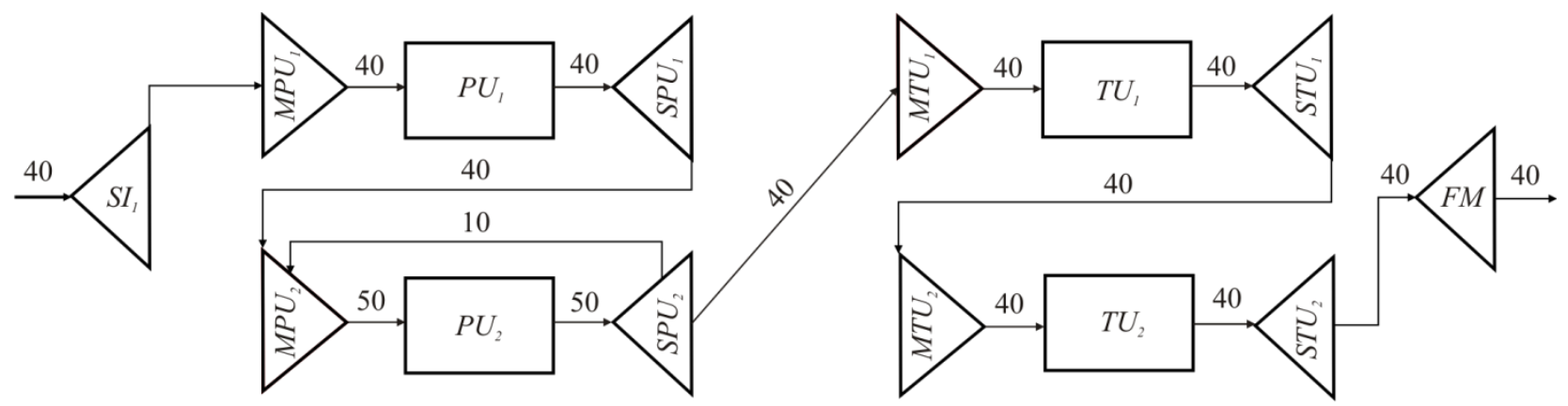

Figure 19. Water network with 6 removable streams and with local recycle.

As can be seen from Table 5 the total network cost for the option without local recycle is a little higher compared to the case with local recycle. The main reason is the higher wastewater flowrate $(50 \mathrm{t} / \mathrm{h})$ which must be treated in treatment unit $\mathrm{TU}_{2}$.

\subsection{Example 2}

The main goal of this example is to demonstrate the capability of the proposed model to solve water network problems of different complexity to global optimality and to compare results with the ones reported in the literature. Data for this example is taken from the literature (Example 1-4 given by Karuppiah and Grossmann, 2006). The relative optimality tolerance in all examples was set to 0.01. Here we used the general purpose optimization software BARON (Sahinidis, 1996) to solve all the problems. The optimization results reported in the paper given by Karuppiah and Grossmann (2006) and the results obtained with the proposed model in this paper are shown in Table 6. 
Table 6. Comparison of optimization results for NLP problems different complexity.

\begin{tabular}{|c|c|c|c|c|c|}
\hline \multirow[b]{2}{*}{ Problem } & \multirow[b]{2}{*}{ No units } & \multicolumn{2}{|c|}{ (Karuppiah and Grossmann, 2006) } & \multicolumn{2}{|c|}{ Proposed NLP method } \\
\hline & & Global optimum & Total time (s) & Global optimum & Total time (s) \\
\hline 1 & 2PU-2TU & $117.05 \mathrm{t} / \mathrm{h}$ & 37.72 & 101.57 & 0.36 \\
\hline 2 & 3PU-3TU & $\$ 381,751.35$ & 13.21 & $\$ 381,751.35$ & 0.34 \\
\hline 3 & 4PU-2TU & $\$ 874,057.37$ & 0.90 & $\$ 874,057.37$ & 0.11 \\
\hline 4 & 5PU-3TU & $\$ 1,033,810.95$ & 231.37 & $\$ 1,033,810.95$ & 16.15 \\
\hline
\end{tabular}

The first problem involves a water network with 2 process units (PU) and 2 treatment (TU) units. The objective function was to minimize the total sum of the freshwater consumption and total flowrate of wastewater treated inside of treatment units (Eq. 40). Karuppiah and Grossmann (2006) reported the optimal solution $117.05 \mathrm{t} / \mathrm{h}$. However, they did not consider local recycle around the process unit which leads to a $13 \%$ reduction in the objective function as seen in Table 6.

In addition, as can be also seen in Table 6, we solved water network problems with 3 process and 3 treatment units, 4 process and 2 treatment units, 5 process and 3 treatment units. For these examples the values of the objective function (minimum total network cost) obtained by the proposed model in this paper are the same as the ones reported by Karuppiah and Grossmann (2006), while the computational time is smaller in all cases. The main reasons for improved performance are good variable bounds for all flowrates and concentrations, incorporating the cut proposed by Karuppiah and Grossmann (2006) and a faster computer.

\subsection{Example 3}

This example demonstrates the capability of the proposed model to solve water network problems with both mass transfer and non mass transfer operations. The case study, a Specialty Chemical Plant, is taken from Wang and Smith (1995). The process flowsheet is given in Figure 20, which has a consumption of $165 \mathrm{t} / \mathrm{h}$ freshwater. It should be noted that the water entering the process is greater than the wastewater flow in the exit since some water leaves the process with the product. Table 7 gives limiting process data for water-using operations in the process and its utility system. 
Table 7. Limiting process data for Specialty Chemical Plant (Wang and Smith, 1995).

\begin{tabular}{lcccc}
\hline Operation & $\begin{array}{c}\text { Water in } \\
(\mathrm{t} / \mathrm{h})\end{array}$ & $\begin{array}{c}\text { Water out } \\
(\mathrm{t} / \mathrm{h})\end{array}$ & $\begin{array}{c}c_{\text {in }} \\
(\mathrm{ppm})\end{array}$ & $\begin{array}{c}c_{\text {out }} \\
(\mathrm{ppm})\end{array}$ \\
\hline Reactor & 80 & 20 & 100 & 1,000 \\
Cyclone & 50 & 50 & 200 & 700 \\
Filtration & 10 & 40 & 0 & 100 \\
Steam System & 10 & 10 & 0 & 10 \\
Cooling System & 15 & 5 & 10 & 100 \\
\hline
\end{tabular}

Water-using operations such as the reactor, filtration and cooling tower have different water flowrates at their inlets and outlets. In the case of the reactor and the cooling tower there are losses of water. However, in the case of filtration process there is gain of water. Water flowrates of these operations can be divided in two parts. The first part is considered to be unchanged through the process, while the second part involves loss or gain of water (Wang and Smith, 1995). According to this, the modified process data for this example are given in Table 8. 


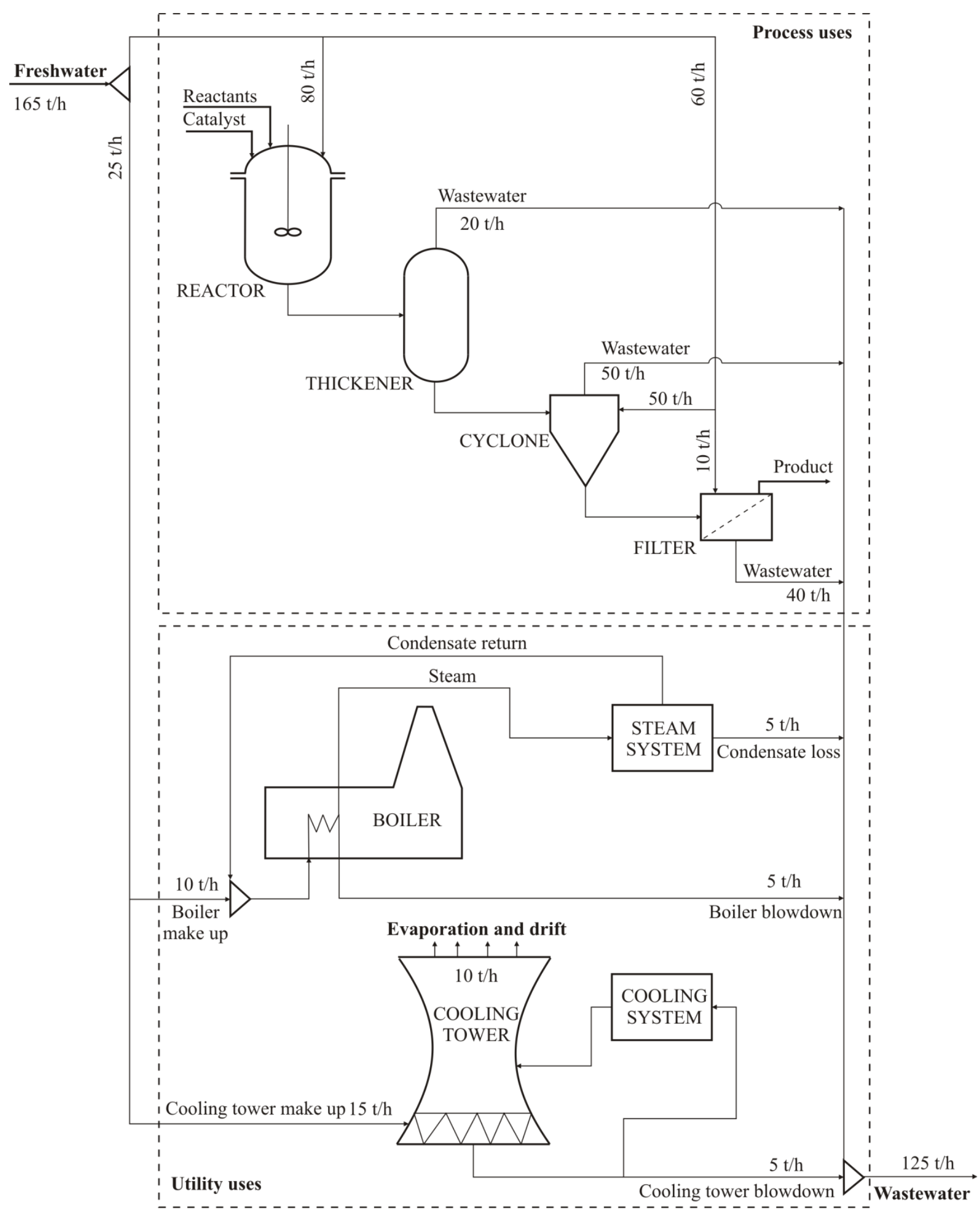

Figure 20. Flowsheet for Specialty Chemical Plant with its utility systems. 
Table 8. Modified limiting process data for Specialty Chemical Plant.

\begin{tabular}{lcccc}
\hline Operation & $\begin{array}{c}\text { Water in } \\
(\mathrm{t} / \mathrm{h})\end{array}$ & $\begin{array}{c}\text { Water out } \\
(\mathrm{t} / \mathrm{h})\end{array}$ & $\begin{array}{c}c_{\text {in }} \\
(\mathrm{ppm})\end{array}$ & $\begin{array}{c}c_{\text {out }} \\
(\mathrm{ppm})\end{array}$ \\
\hline Reactor I & 20 & 20 & 100 & 1,000 \\
Reactor II & 60 & 0 & 100 & - \\
Cyclone & 50 & 50 & 200 & 700 \\
Filtration I & 10 & 10 & 0 & 100 \\
Filtration II & 0 & 30 & - & 100 \\
Steam System & 10 & 10 & 0 & 10 \\
Cooling System I & 5 & 5 & 10 & 100 \\
Cooling System II & 10 & 0 & 10 & - \\
\hline
\end{tabular}

According to the flowsheet in Figure 20, the operations that can be considered as the water sink/demand units are reactor and cooling tower. The water source unit is filtration. Representation of these units and their flowrates is shown in Figures 21 and 22, respectively.

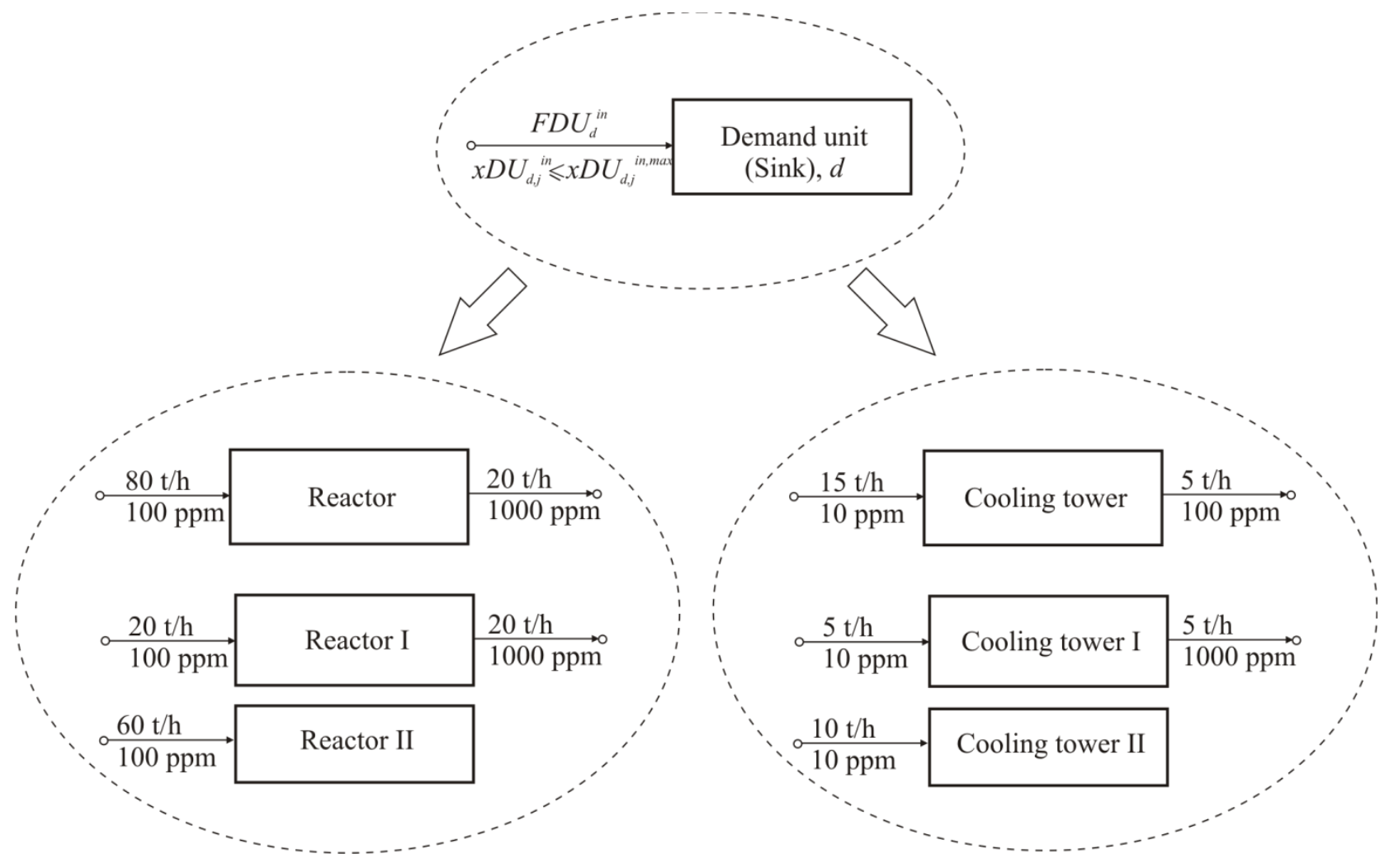

Figure 21. Representation of flowrates of the water demand unit. 


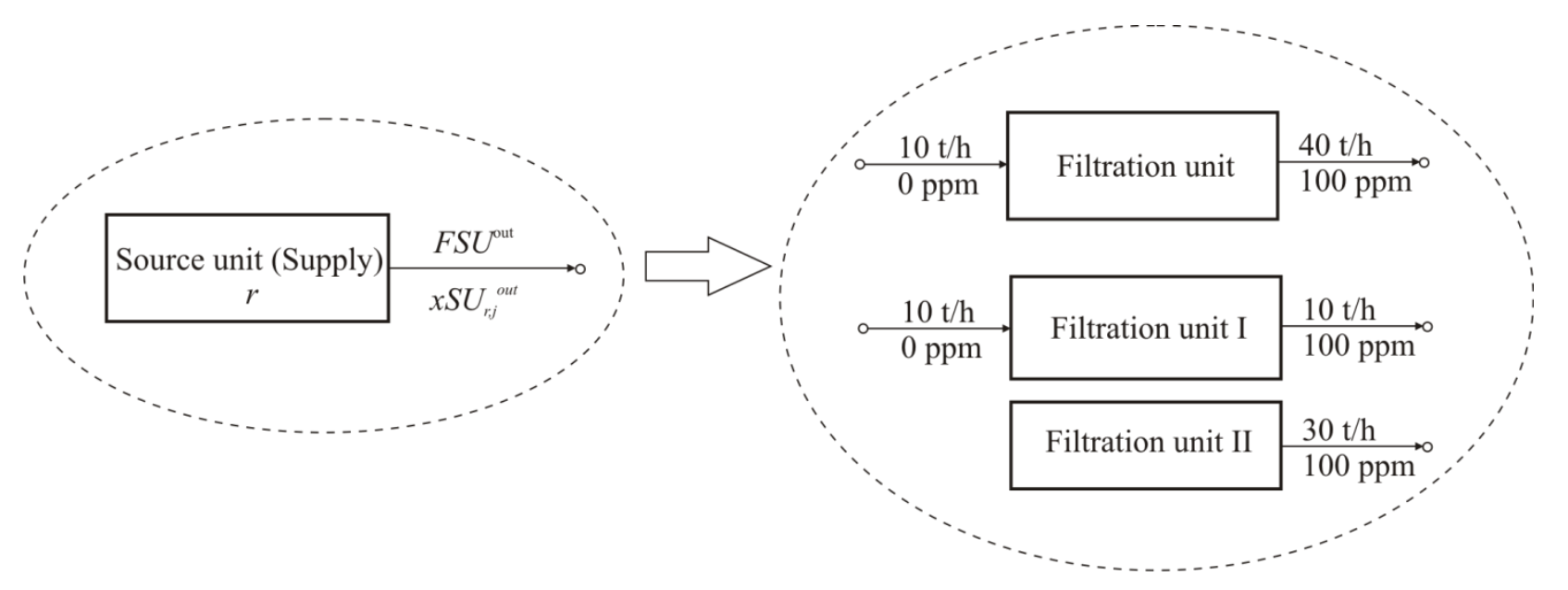

Figure 22. Representation of flowrates of the water source unit.

The optimal solution of the water network with reuse for the data in Table 8 is given in Figure 23 . The optimization was performed with the global optimization BARON solver and the selected optimality tolerance was zero. The objective function was to minimize the total consumption of freshwater for network. The new water network design yields a reduction in freshwater consumption of about $45 \%$ (from $165 \mathrm{t} / \mathrm{h}$ to $90.64 \mathrm{t} / \mathrm{h}$ ) and wastewater generation of about $59 \%$ (from $125 \mathrm{t} / \mathrm{h}$ to $50.64 \mathrm{t} / \mathrm{h})$. It is worth to mention that the values of water consumption and wastewater generation are the same as the ones reported by Wang and Smith (1995) and Bandyopadhyay, Ghanekar, and Pillai (2006). 


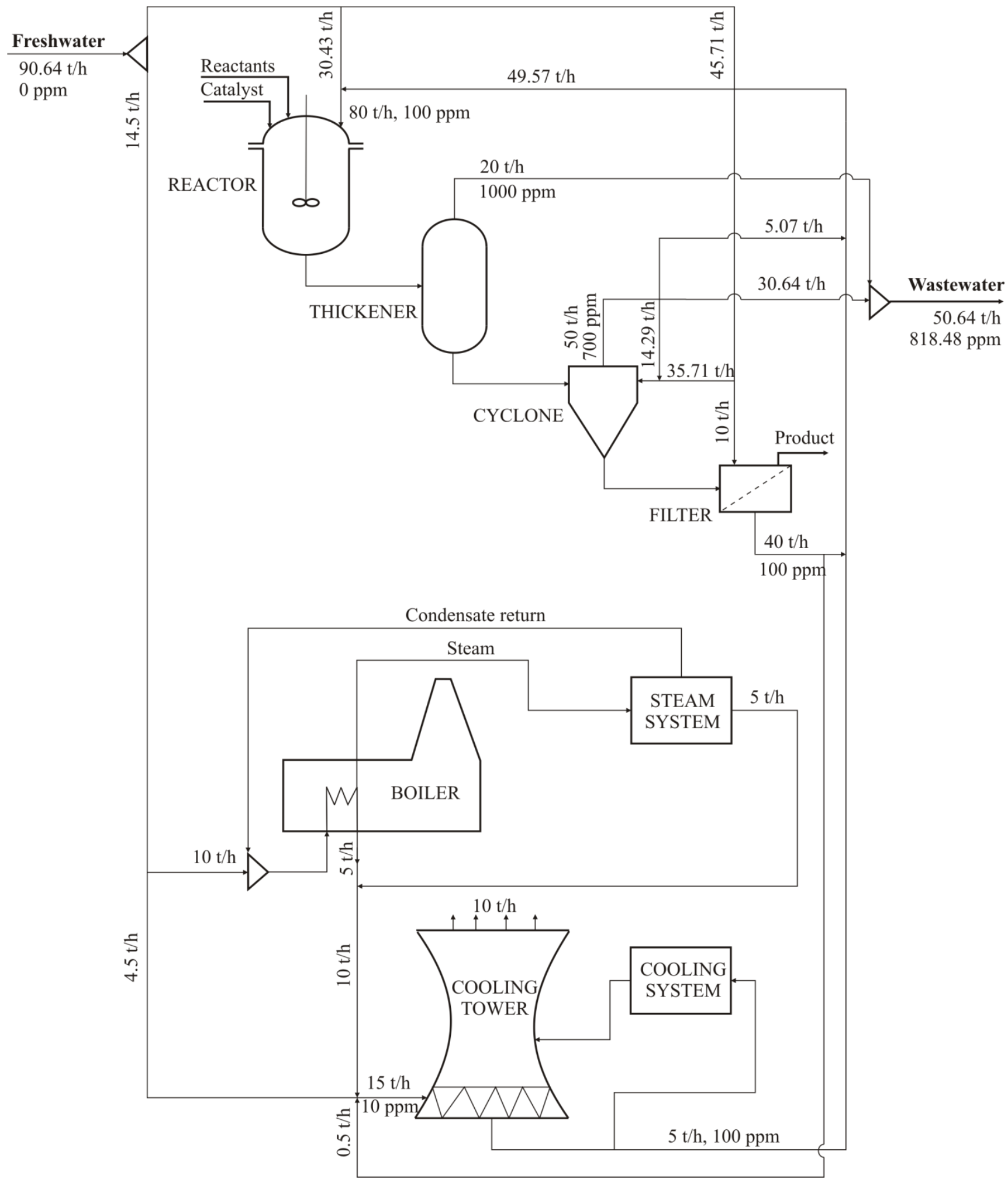

Figure 23. Optimal design of water network for Specialty Chemical Plant. 
In addition to this, Bandyopadhyay, Ghanekar, and Pillai (2006) solved the same problem using their proposed method for targeting minimum effluent treatment flowrate satisfying the minimum freshwater requirement. In their paper the water allocation network incorporates two treatment units. They assumed the \% removal for each contaminant in treatment units to be 90 , and maximum allowable concentration of contaminants in the discharge effluent to the environment to be $50 \mathrm{ppm}$. We solved the same problem by sequential optimization of water-using and water treatment units. The objective function for optimization of water using operations is to minimize the sum of freshwater consumption and the objective function for optimization of treatment operations is to minimize the sum of water flowrates going to treatment units. The optimal network design is the same as reported by Bandyopadhyay, Ghanekar, and Pillai (2006) (freshwater consumption of 90.64 $\mathrm{t} / \mathrm{h}$ ). However, we also optimized simultaneously the same problem as an integrated network with the water-using operations and water treatment operations. We assumed to have two treatment units with the same \% removal for contaminant in the treatment units (90\%). Also, we considered the options with/without local recycle around process units. In both cases the new design yields a reduction in freshwater consumption of about $73 \%$ (from $165 \mathrm{t} / \mathrm{h}$ to $45 \mathrm{t} / \mathrm{h}$ ) and wastewater generation of about 96\% (from $125 \mathrm{t} / \mathrm{h}$ to $5 \mathrm{t} / \mathrm{h}$ ) compared to the base case. Moreover, we assumed to have two treatment units for wastewater treatment, but only one is selected by the optimization. The optimal solution of the water network design with recycle around process unit is given in Figure 24. 


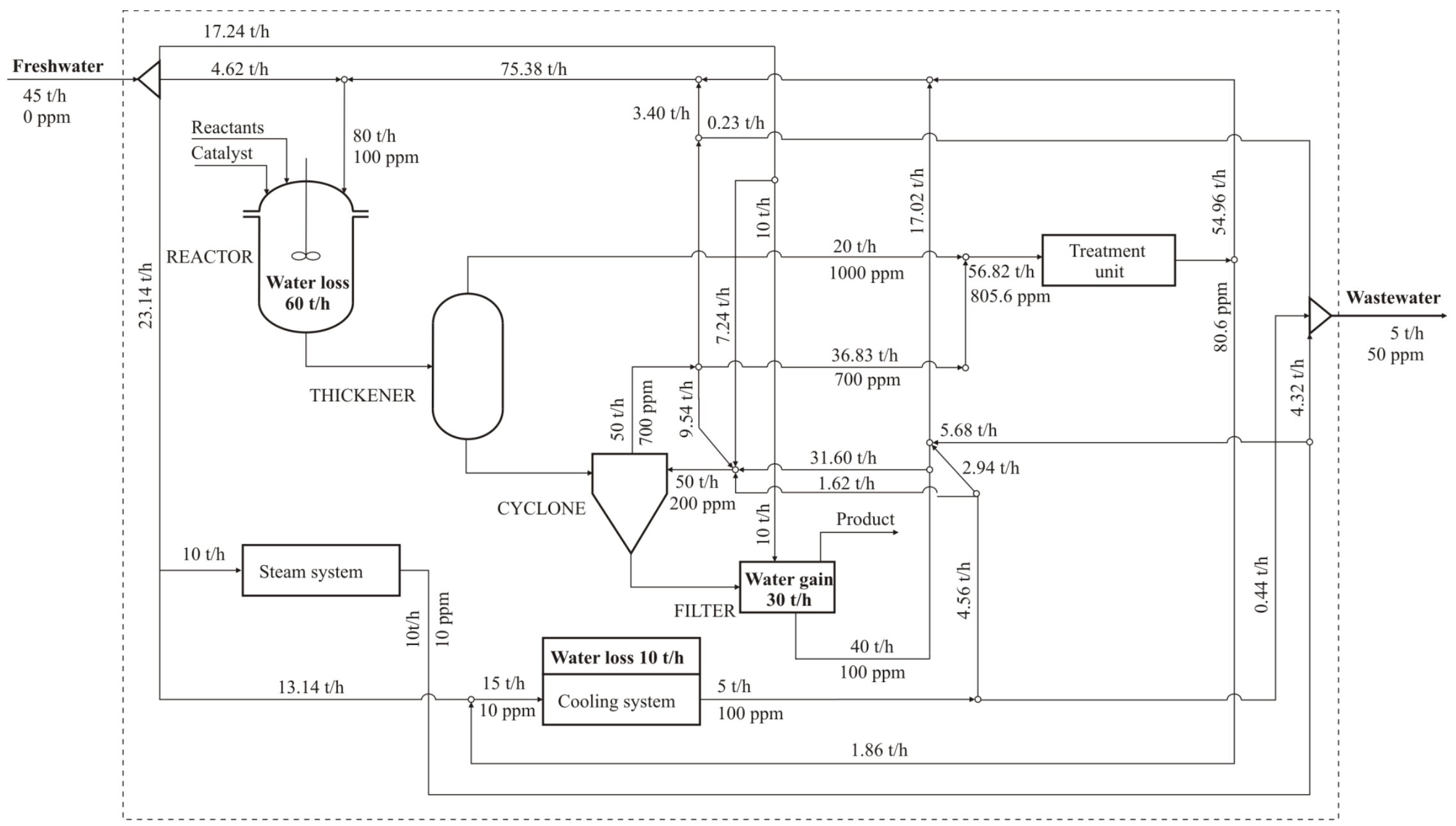

Figure 24. Optimal design of water network for Specialty Chemical Plant by simultaneous optimization with local recycle. 


\subsection{Example 4}

In the process industry water-using operations can have different maximum allowed concentrations at their inlets. Therefore, water sources of different quality can be used to satisfy water-using concentration and flowrate demands. The higher quality water is more expensive than the lower quality water. The objective of this example is to illustrate that the proposed method can be applied to a complex industrial water network consisting of four sources of water, six water-using operations, three water treatment operations and three contaminants. Data for water sources, water-using operations, and treatment units are given in Tables 9, 10 and 11.

Table 9. Data for water sources for Example 4.

\begin{tabular}{|c|c|c|c|c|}
\hline \multirow[t]{2}{*}{$\begin{array}{l}\text { Water } \\
\text { source }\end{array}$} & \multirow[t]{2}{*}{$\begin{array}{l}\text { Cost of water } \\
\text { source }(\$ / t)\end{array}$} & \multicolumn{3}{|c|}{$\begin{array}{c}\text { Concentration of } \\
\text { contaminants (ppm) }\end{array}$} \\
\hline & & A & B & $\mathrm{C}$ \\
\hline $\mathrm{SW}_{1}$ & 1.00 & 0 & 0 & 0 \\
\hline $\mathrm{SW}_{2}$ & 0.50 & 25 & 35 & 35 \\
\hline $\mathrm{SW}_{3}$ & 0.20 & 45 & 40 & 40 \\
\hline $\mathrm{SW}_{4}$ & 0.15 & 50 & 50 & 50 \\
\hline
\end{tabular}

Table 10. Data for process units for Example 4.

\begin{tabular}{cccccccc}
\hline $\begin{array}{c}\text { Process } \\
\text { unit }\end{array}$ & $\begin{array}{c}\text { Flowrate } \\
(\mathrm{t} / \mathrm{h})\end{array}$ & \multicolumn{3}{c}{$\begin{array}{c}\text { Discharge load } \\
(\mathrm{kg} / \mathrm{h})\end{array}$} & \multicolumn{3}{c}{$\begin{array}{c}\text { Maximum inlet concentration } \\
(\mathrm{ppm})\end{array}$} \\
\cline { 3 - 8 } & & $\mathrm{A}$ & $\mathrm{B}$ & $\mathrm{C}$ & $\mathrm{A}$ & $\mathrm{B}$ & $\mathrm{C}$ \\
\hline $\mathrm{PU}_{1}$ & 40 & 1 & 1.5 & 1 & 25 & 25 & 25 \\
$\mathrm{PU}_{2}$ & 50 & 1 & 1 & 1 & 50 & 50 & 50 \\
$\mathrm{PU}_{3}$ & 60 & 1 & 1 & 1 & 50 & 50 & 50 \\
$\mathrm{PU}_{4}$ & 70 & 2 & 2 & 2 & 50 & 50 & 50 \\
$\mathrm{PU}_{5}$ & 80 & 1 & 1 & 0 & 25 & 25 & 25 \\
$\mathrm{PU}_{6}$ & 90 & 1 & 1 & 0 & 10 & 10 & 10 \\
\hline
\end{tabular}


Table 11. Data for treatment units for Example 4.

\begin{tabular}{|c|c|c|c|c|c|c|}
\hline \multirow[t]{2}{*}{ Unit } & \multicolumn{3}{|c|}{$\begin{array}{c}\% \text { removal for } \\
\text { contaminant }\end{array}$} & \multirow[t]{2}{*}{$\begin{array}{l}\text { IC (Investment } \\
\text { cost coefficient) }\end{array}$} & \multirow[t]{2}{*}{$\begin{array}{l}\text { OC (Operating } \\
\text { cost coefficient) }\end{array}$} & \multirow[t]{2}{*}{$\alpha$} \\
\hline & A & B & $\mathrm{C}$ & & & \\
\hline $\mathrm{TU}_{1}$ & 95 & 0 & 0 & 16,800 & 1 & 0.7 \\
\hline $\mathrm{TU}_{2}$ & 0 & 0 & 95 & 9,500 & 0.04 & 0.7 \\
\hline $\mathrm{TU}_{3}$ & 0 & 95 & 0 & 12,600 & 0.0067 & 0.7 \\
\hline
\end{tabular}

Data for the process and treatment units are taken from Karuppiah and Grossmann (2006) and are slightly modified for this example. The annualized factor for investment of treatment units, the total time for the network plant operation, and maximum allowable contaminants concentration in the discharge effluent to the environment are the same as in the previous example. The objective function is to minimize the total network cost. The optimality tolerance selected for the optimization was 0.05 with the global optimization solver BARON. The total cost for the network is $\$ 1,149,710.83 /$ year, and the optimal solution of the water network design is given in Figure 25. 


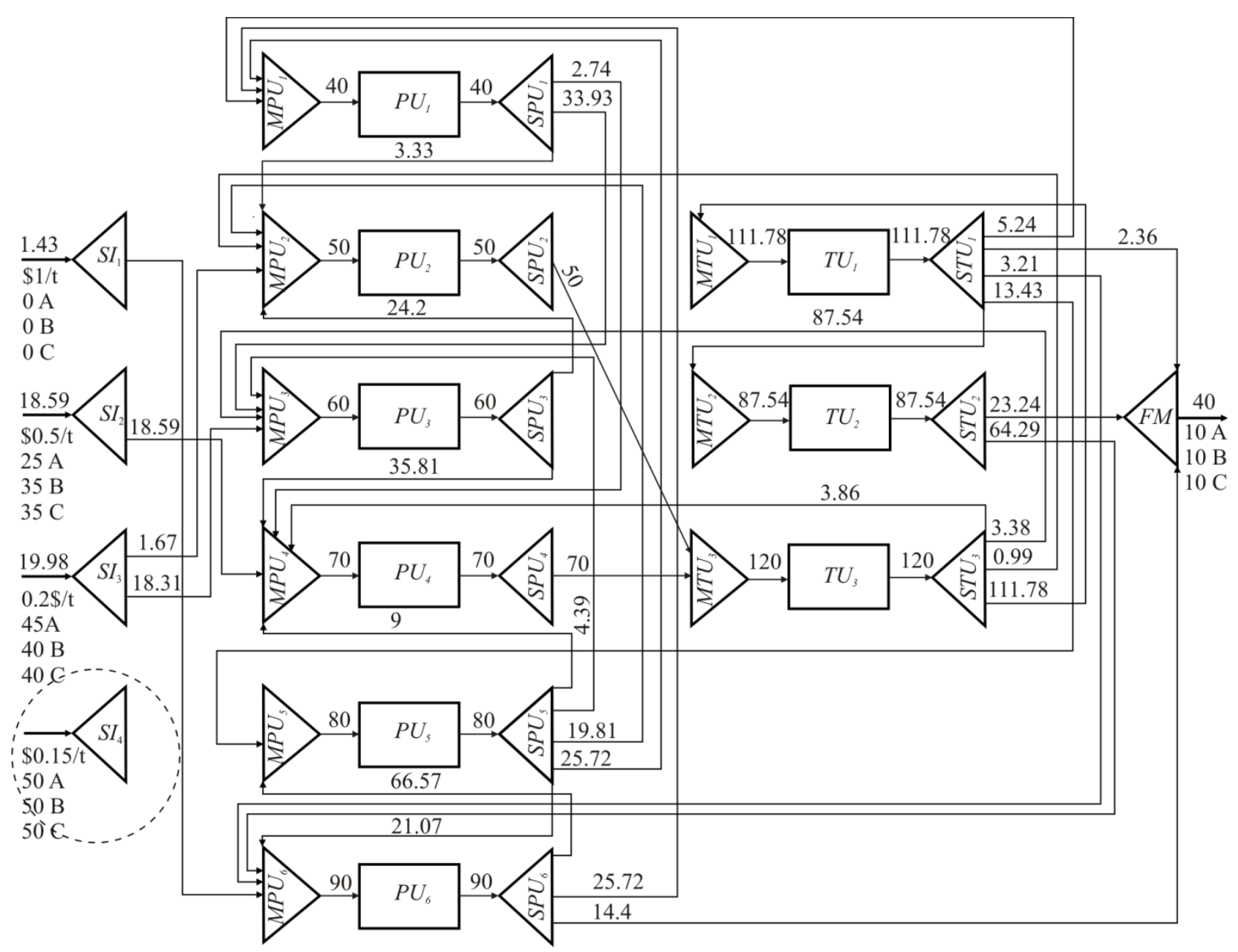

Figure 25. Optimal design of the integrated process network for Example 4.

It should be noticed that the freshwater source 4 is not selected. In addition, pure water from source 1, which has the highest cost, is minimized at the expense of using more of the lower quality water.

\subsection{Example 5}

The objective of this example is to illustrate the application of the proposed MINLP solution method on a large-scale industrial network consisting of five water-using units, three wastewater treatment units and three contaminants (A, B, C). Data for this example are given in Tables 12 and 13 (Karuppiah and Grossmann 2006). The water network superstructure is given in Figure 26. It includes all feasible connections between units in the network. 
Table 12. Data for s for Example 5.

\begin{tabular}{|c|c|c|c|c|c|c|c|}
\hline \multirow[t]{2}{*}{$\begin{array}{l}\text { Process } \\
\text { unit }\end{array}$} & \multirow[t]{2}{*}{$\begin{array}{l}\text { Flowrate } \\
(\mathrm{t} / \mathrm{h})\end{array}$} & \multicolumn{3}{|c|}{$\begin{array}{l}\text { Discharge load } \\
(\mathrm{kg} / \mathrm{h})\end{array}$} & \multicolumn{3}{|c|}{$\begin{array}{l}\text { Maximum inlet } \\
\text { concentration (ppm) }\end{array}$} \\
\hline & & A & B & C & A & B & C \\
\hline $\mathrm{PU}_{1}$ & 40 & 1 & 1.5 & 1 & 0 & 0 & 0 \\
\hline $\mathrm{PU}_{2}$ & 50 & 1 & 1 & 1 & 50 & 50 & 50 \\
\hline $\mathrm{PU}_{3}$ & 60 & 1 & 1 & 1 & 50 & 50 & 50 \\
\hline $\mathrm{PU}_{4}$ & 70 & 2 & 2 & 2 & 50 & 50 & 50 \\
\hline $\mathrm{PU}_{5}$ & 80 & 1 & 1 & 0 & 25 & 25 & 25 \\
\hline
\end{tabular}

Table 13. Data for treatment units for Example 6.

\begin{tabular}{|c|c|c|c|c|c|c|}
\hline \multirow[t]{2}{*}{$\begin{array}{l}\text { Uni } \\
\mathrm{t}\end{array}$} & \multicolumn{3}{|c|}{$\begin{array}{c}\% \text { removal for } \\
\text { contaminant }\end{array}$} & \multirow[t]{2}{*}{$\begin{array}{l}\text { IC (Investment } \\
\text { cost coefficient) }\end{array}$} & \multirow[t]{2}{*}{$\begin{array}{l}\text { OC (Operating } \\
\text { cost coefficient) }\end{array}$} & \multirow[t]{2}{*}{$\alpha$} \\
\hline & $\mathrm{A}$ & B & $\mathrm{C}$ & & & \\
\hline $\mathrm{TU}_{1}$ & 95 & 0 & 0 & 16,800 & 1 & 0.7 \\
\hline $\mathrm{TU}_{2}$ & 0 & 0 & 95 & 9,500 & 0.04 & 0.7 \\
\hline $\mathrm{TU}_{3}$ & 0 & 95 & 0 & 12,600 & 0.0067 & 0.7 \\
\hline
\end{tabular}

The annualized factor for investment of the treatment units, the total time for the network plant operation, and maximum allowable contaminants concentration in the discharge effluent to the environment are the same as in the previous example. The objective function is to minimize the total network cost. We used the BARON and LINDOGlobal solvers in this example. 


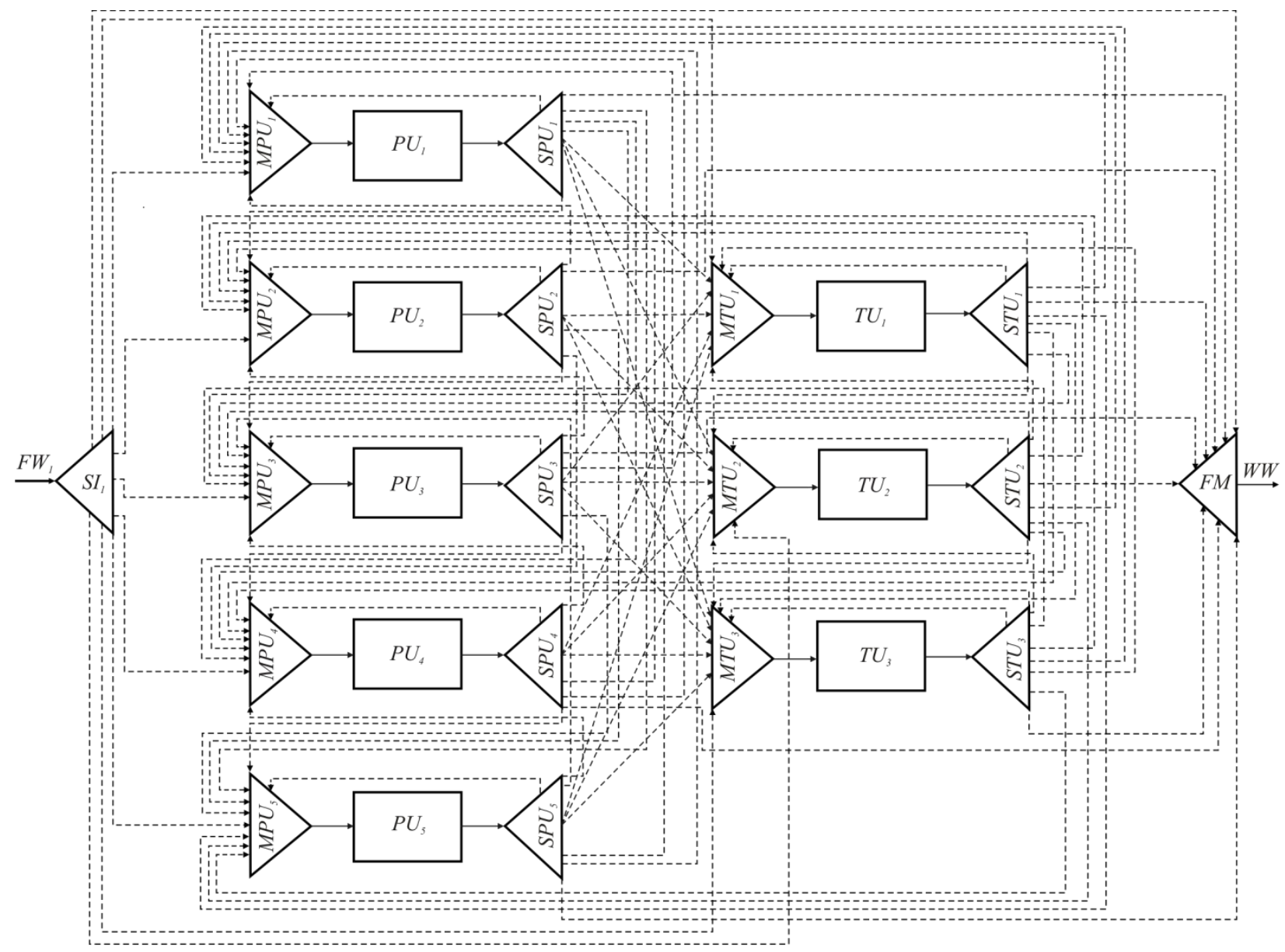

Figure 26. Water network superstructure for Example 5.

The MINLP model for this example is solved using the proposed solution approach. In the first step, we solved the NLP model of the network shown in Figure 26 and obtained the global solution. Then, we fixed all zero flowrates, and update bounds before solution of reduced the MINLP. The total network cost of the MINLP model was $\$ 1,062,700.53 /$ year and the number of removable piping connections in the network was 22. In order to control the piping complexity we restricted the number of connections in the network and solved the corresponding MINLP problems. Results of the optimization are given in Figure 27, which shows the costs of the water networks for different number of removable connections. It is interesting to note that the freshwater consumption in all cases was the same (40 t/h). In addition, it should be mentioned that the greatly simplified water network shown in Figure 28 has 13 removable connections, and the total network cost $\$ 1,223,698.7 /$ year which is about $15 \%$ increase in the value of objective function compared to the optimal base case $(\$ 1,062,700.5 /$ year $)$ with 22 removable connections. 


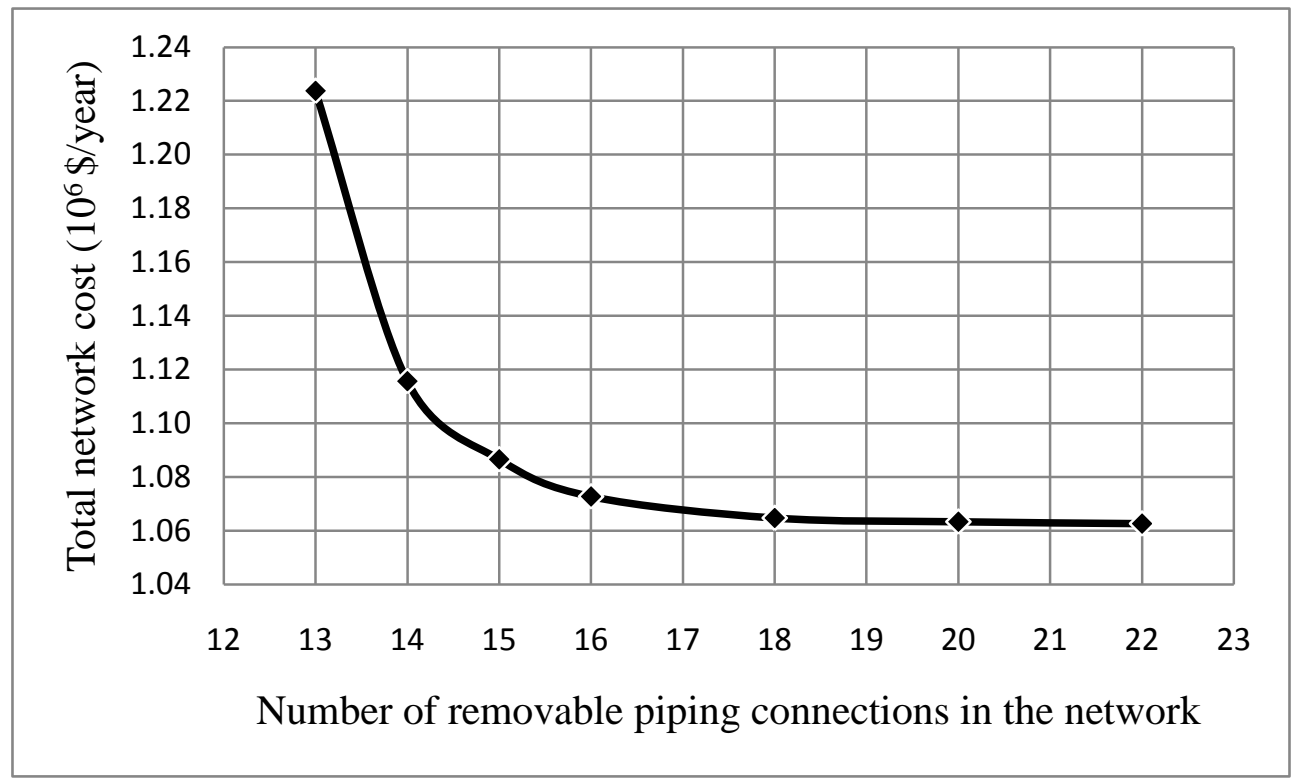

Figure 27. The costs of the water networks for different number of removable connections.

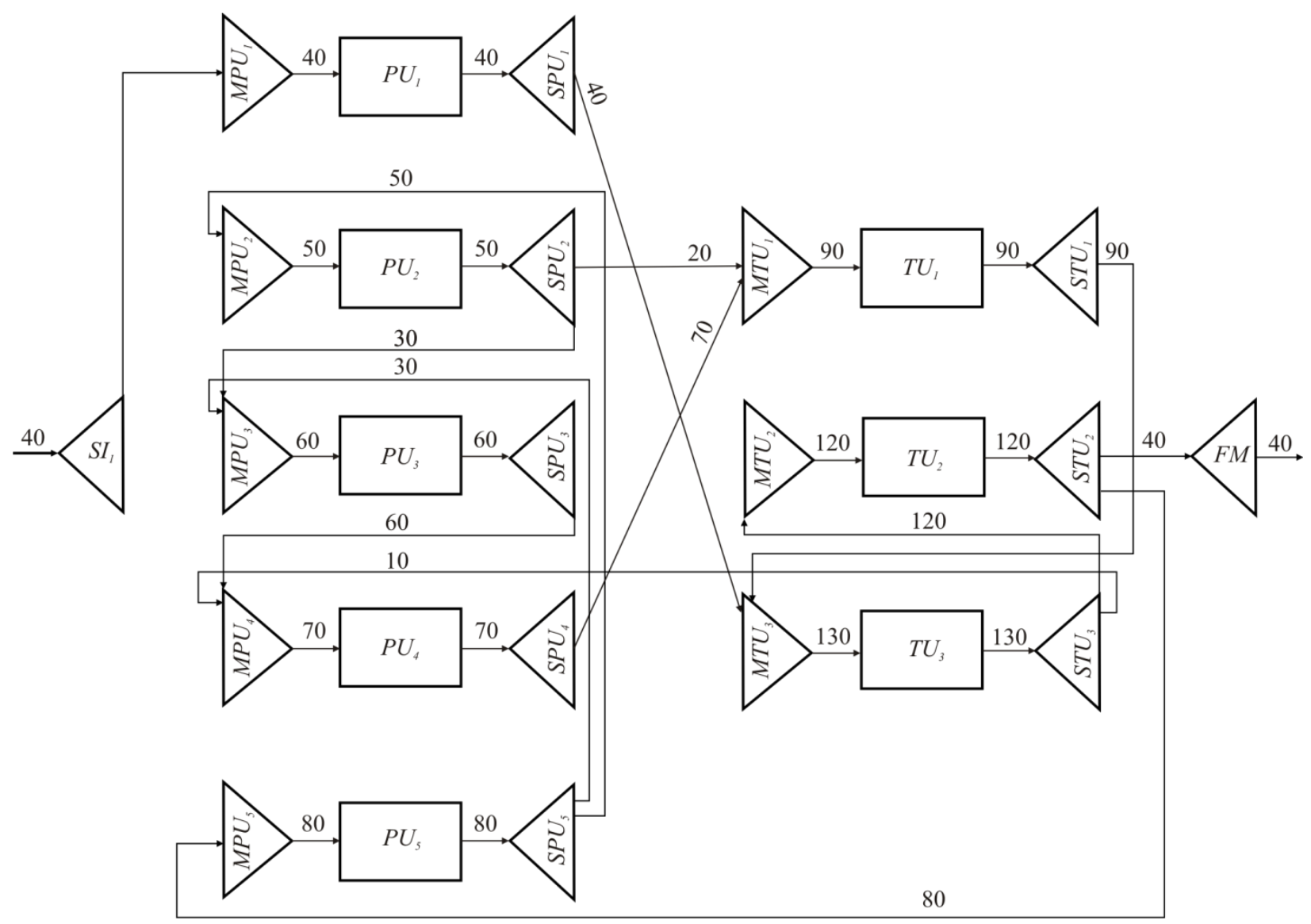

Figure 28. Optimal design of the simplified water network with 13 removable connections. 


\subsection{Example 6}

This example illustrates the different possibilities for reducing of the water consumption and the total costs for the network consists of the water pre-treatment subsystem, the water-using subsystem, and the wastewater treatment subsystem. Moreover, we present results of the complete water integration, and zero liquid discharge cycles when all feasible interconnections between previously mentioned subsystems are allowed in the network.

Table 14 shows the data for this example that involves two process units $\left(\mathrm{PU}_{1}, \mathrm{PU}_{2}\right)$, two water pre-treatment units $\left(\mathrm{TU}_{1}, \mathrm{TU}_{2}\right)$, one wastewater treatment unit $\left(\mathrm{TU}_{3}\right)$, and four contaminants.

Table 14. Data of process units.

\begin{tabular}{llllllllll}
\hline Unit & $\begin{array}{l}\text { Flowrate } \\
(\mathrm{t} / \mathrm{h})\end{array}$ & \multicolumn{3}{l}{$\begin{array}{l}\text { Discharge load } \\
(\mathrm{kg} / \mathrm{h})\end{array}$} & & \multicolumn{4}{l}{$\begin{array}{l}\text { Maximum inlet } \\
\text { concentration }(\mathrm{ppm})\end{array}$} \\
\cline { 2 - 11 } & & $\mathrm{A}$ & $\mathrm{B}$ & $\mathrm{C}$ & $\mathrm{D}$ & $\mathrm{A}$ & $\mathrm{B}$ & $\mathrm{C}$ & $\mathrm{D}$ \\
$\mathrm{PU}_{1}$ & 40 & 1 & 1.5 & 1 & 1 & 0 & 0 & 0 & 0 \\
$\mathrm{PU}_{2}$ & 50 & 1 & 1 & 1 & 1 & 50 & 50 & 50 & 50 \\
\hline
\end{tabular}

Data for the operating cost and the investment cost of the water pre-treatment $\left(\mathrm{TU}_{1}\right.$ and $\left.\mathrm{TU}_{2}\right)$ and the wastewater treatment $\left(\mathrm{TU}_{3}\right)$ units are given in Table 15 and they are taken from Faria and Bagajewicz (2009).

Table 15. Data of pre-treatment and treatment units.

\begin{tabular}{lccc}
\hline Unit & $\begin{array}{c}\text { IC (Investment } \\
\text { cost coefficient) }\end{array}$ & $\begin{array}{c}\text { OC (Operating } \\
\text { cost coefficient) }\end{array}$ & $\alpha$ \\
\cline { 2 - 4 } & & & \\
\hline $\mathrm{TU}_{1}$ (Pre-treatment 1) & 10,000 & 0.10 & 0.7 \\
$\mathrm{TU}_{2}$ (Pre-treatment 2) & 25,000 & 1.15 & 0.7 \\
$\mathrm{TU}_{3}$ (Wastewater treatment) & 30,000 & 1.80 & 0.7 \\
\hline
\end{tabular}

There is one freshwater source with four contaminants (100 ppm A, 100 ppm B, 100 ppm C, and $100 \mathrm{ppm} \mathrm{D}$ ). The freshwater cost is assumed to be $\$ 0.1 /$ ton. The annualized factor for investment of the treatment units is assumed to be 0.1 , and the total time for the network operation in a year $8600 \mathrm{~h}$. The environmental discharge limit for all contaminants (A, B, C, and 
D) concentration is $10 \mathrm{ppm}$. In addition, the maximum inlet contaminants concentration of the water pre-treatment unit $\mathrm{TU}_{1}$ is $100 \mathrm{ppm}$ and $\mathrm{TU}_{2} 10 \mathrm{ppm}$. We assume that the water pretreatment units can purify the freshwater to the water quality down to $10 \mathrm{ppm}$ for each contaminant $\left(\mathrm{TU}_{1}\right)$ and down to $0 \mathrm{ppm}\left(\mathrm{TU}_{2}\right)$. The percent removal for contaminants in the wastewater treatment unit $\left(\mathrm{TU}_{3}\right)$ was $95 \%$. We used the BARON to solve the MINLP in this example.

Figure 29 shows the optimal design of water network when the recycling water from the water-using subsystem and the wastewater treatment subsystem to the water pre-treatment subsystem is not allowed. The freshwater consumption is $50 \mathrm{t} / \mathrm{h}$ and the total network cost $\$ 1,244,299.7$ /year.

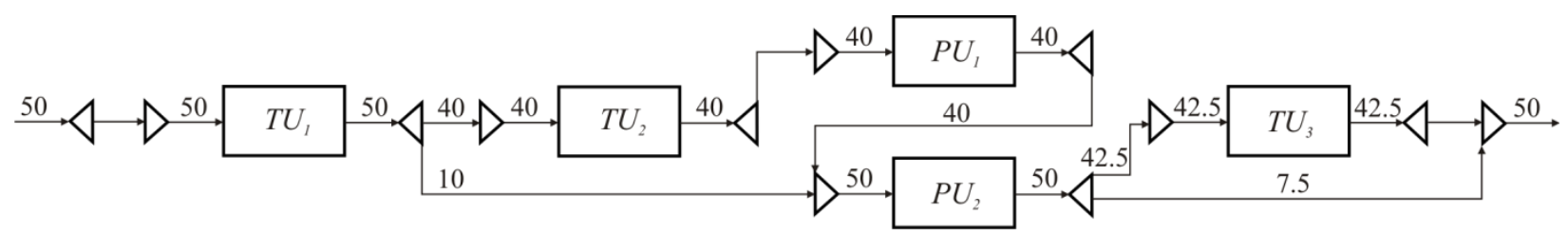

Figure 29. Recycling water from the water-using /wastewater treatment subsystem to the water pre-treatment subsystem is not allowed

In the next case shown in Figure 30, we assume that recycling water from the water-using and the wastewater treatment subsystem to the water pre-treatment subsystem is allowed. Note that the freshwater consumption is reduced to $40 \mathrm{t} / \mathrm{h}$ compared to the previous case. In addition, the total network cost is lower, $\$ 1,223,838.0 /$ year.

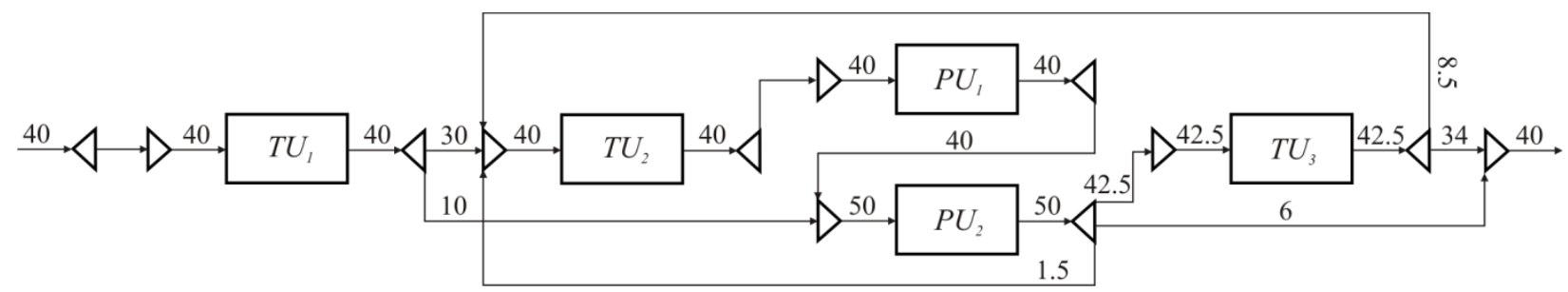

Figure 30. Recycling water from the water-using /wastewater treatment subsystem to the water pre-treatment subsystem is allowed

As can be seen from Figure 30, the network has the recycles from the water-using unit 2 (1.5 $\mathrm{t} / \mathrm{h})$ and from the wastewater treatment unit $3(8.5 \mathrm{t} / \mathrm{h})$ to the water pre-treatment unit 2. In addition, both types of freshwater (10 ppm and $0 \mathrm{ppm})$ are used in the network. 
The optimal design of water network with local recycle is shown in Figure 31. The total network cost is $\$ 1,057,659.3 /$ year and the freshwater consumption is the same $(40 \mathrm{t} / \mathrm{h})$ as in Figure 30, while the wastewater flowrate of treatment unit 3 is reduced from $42.5 \mathrm{t} / \mathrm{h}$ to $32.28 \mathrm{t} / \mathrm{h}$.

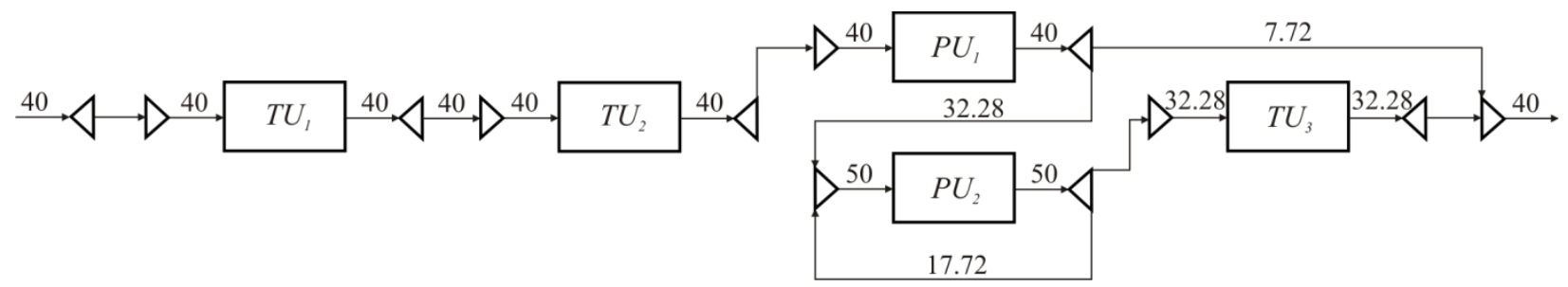

Figure 31. Optimal design of water network with local recycle.

It is worth pointing out that water networks can have zero liquid discharge cycles when all connections between the subsystems in the network are allowed (the complete water integration). In Figure 32, we present the optimal network designs with zero liquid discharge cycles for this example. The total network cost for option without local recycle is $\$ 1,157,863.5 /$ year and for option with local recycle $\$ 971,320.7 /$ year. Note that we assumed to have two pre-treatment units, but just one $\left(\mathrm{TU}_{2}\right)$ is selected by the optimization. Note also that although the use of freshwater has been eliminated the cost of treatment in Figure 32a is higher due to its higher flowrate $(43.77 \mathrm{t} / \mathrm{h}$ vs $32.28 \mathrm{t} / \mathrm{h})$.

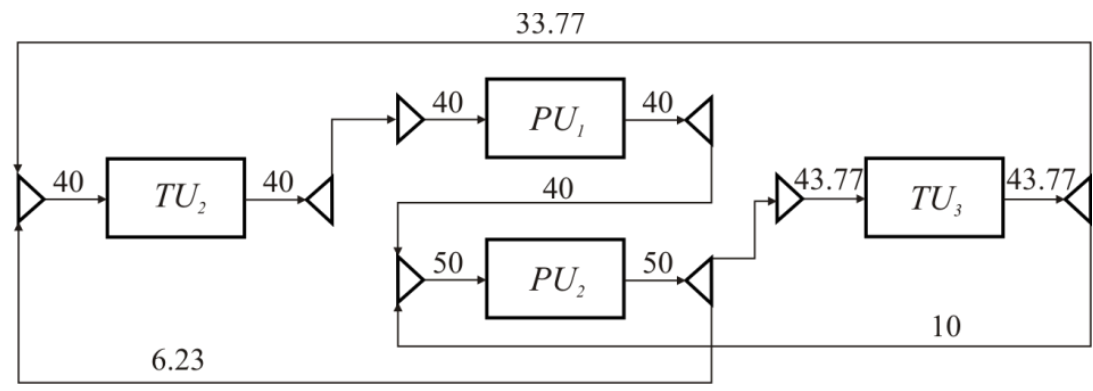

a) without local recycle

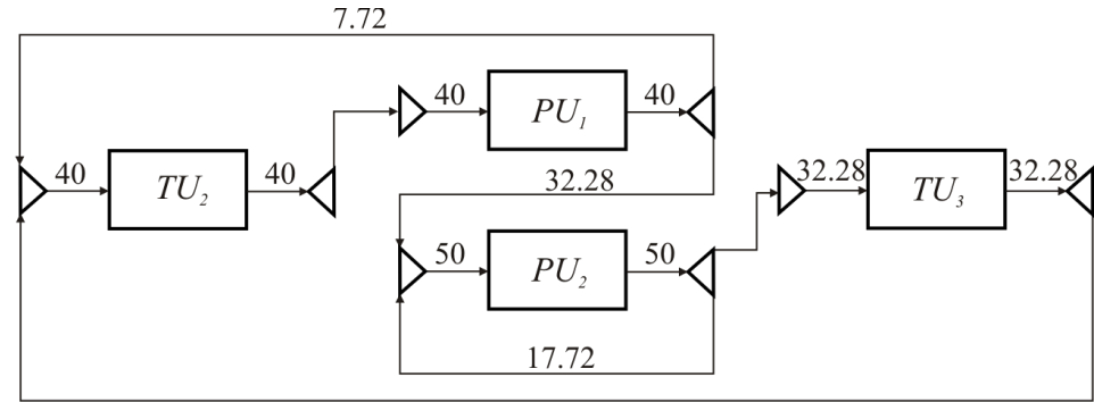

b) with local recycle

Figure 32. Optimal design of water network with zero liquid discharge cycles. 


\section{Model sizes and computational times for Examples 1-6}

Table 16 shows the sizes and computational times for the examples presented in the previous section. As can be seen from this table we solved NLP and MINLP problems of different sizes and complexity. The optimality tolerance selected for optimization in the first, third, fifth, and sixth example was 0.0 and in the second and fourth 0.01 and 0.05. All MINLP examples shown in Table 16 are solved with the proposed two-stage solution. To solve the water network examples to global optimality we used the BARON and LINDOGlobal solvers. The computational times, for problems with no constraints on the removable streams were reasonable in all cases (see Table 16), although, it should be mentioned that by increasing size of the MINLP problems computational time increases and in these cases available optimization solvers cannot find an optimal solution in several hours. However, by applying the two-stage strategy as shown in Figure 13 it was possible to solve the corresponding problems in much less time.

Table 16. Model statistics and computational results for Examples 1-6.

\begin{tabular}{|c|c|c|c|c|c|}
\hline Example & Number of units & $\begin{array}{c}\text { Continuous } \\
\text { variables }\end{array}$ & $\begin{array}{l}\text { Discrete } \\
\text { variables }\end{array}$ & Constraints & $\begin{array}{c}\text { CPU time } \\
(\mathrm{s})\end{array}$ \\
\hline \multirow[t]{4}{*}{1} & 2PU-2TU & 55 & - & 44 & $<0.5$ \\
\hline & & $57^{*}$ & - & 44 & $<1.0$ \\
\hline & & 75 & 20 & 85 & $<0.5$ \\
\hline & & $79^{*}$ & 22 & 89 & $<0.5$ \\
\hline \multirow[t]{4}{*}{2} & 2PU-2TU & $57^{*}$ & - & 44 & $<0.5$ \\
\hline & 3PU-3TU & 92 & - & 62 & $<0.5$ \\
\hline & 4PU-2PU & 91 & - & 62 & $<0.5$ \\
\hline & 5PU-3TU & 161 & - & 106 & 16.2 \\
\hline \multirow[t]{3}{*}{3} & $5 \mathrm{PU}$ & 82 & - & 41 & $<0.5$ \\
\hline & 5PU-2TU & 119 & - & 53 & 2.9 \\
\hline & & $126^{*}$ & - & 53 & $<0.5$ \\
\hline \multirow[t]{2}{*}{4} & 6PU-3TU & 223 & - & 121 & 2.0 \\
\hline & & $232^{*}$ & - & 121 & 1.4 \\
\hline 5 & 5PU-3TU & 233 & 72 & 251 & 10.3 \\
\hline \multirow[t]{2}{*}{6} & 2PU-3TU & 135 & 30 & 148 & $<1.0$ \\
\hline & & $145^{*}$ & 35 & 158 & $<1.0$ \\
\hline
\end{tabular}

It should be mentioned that the computational time for Example 5 with no constraints on removable streams (i.e. 22 removable connections) was 10.3 CPUs (BARON) and 56.3 CPUs (LINDOGlobal). We greatly simplified the water network when no more than 13 removable 
connections are used as shown in Figure 28. The computational time for this problem was 153.3 CPUs (BARON) and 159 CPUs (LINDOGlobal). For other limits on the number of removable connections the $(20,18,16,15,14)$ the computational times were significantly higher (up to 600 CPUs (LINDOGlobal)). In addition, the LINDOGlobal solver could not find any solution for 14 removable connections. Finally, it is worth to point that in all other cases of removable connections the optimal solution was the same for both solvers.

\section{Conclusions}

In this paper, we have presented a general superstructure and a global optimization strategy for the design of integrated process water network as well as its separate subsystems. The superstructure includes all feasible interconnections in the network, mass transfer and non-mass transfer water-using processes, wastewater treatment operations, single and multiple sources of water with/without contaminants, and their possible pre-treatment. On the basis of this superstructure, we have formulated nonconvex NLP and MINLP models. The cost of piping and the cost of pumping water are included in the MINLP model. To expedite the global optimization search we have represented the bounds on the variables as general equations obtained by physical inspection of the superstructure. Furthermore, we proposed a two-stage procedure for solving large-scale models.

From the examples it is clear that the proposed approach can be successfully used for solving industrial water network problems as well as for controlling the complexity of water networks. The capabilities of the proposed method were illustrated on several examples, including largescale water network problems. All the examples were solved to global optimality with reasonable computational time using BARON and LINDOGlobal.

\section{Acknowledgments}

The authors would like to express their gratitude to the Fulbright Visiting Scholar Program and the Center for Advanced Process Decision-making, Department of Chemical Engineering at Carnegie Mellon University for support throughout this work. 


\section{Appendix}

Variable bounds for the proposed model

In the proposed NLP/MINLP optimization model the bounds on the variables are represented as general equations in this appendix.

\section{Initial Splitter}

The upper bound of water flowrate at the initial splitter can be determined on the basis of fixed flowrates for all process and demand units.

$$
F W_{s}^{U}=\sum_{p \in P U} F P U_{p}^{i n}+\sum_{d \in D U} F D U_{d}^{i n} \quad \forall s \in S W,|p| \neq 0
$$

The lower bound of the water flowrate at the initial splitter has a minimum fixed flowrate for a process unit if one external source of water exists in the network. If there are more sources of water in the network, the lower bound is set to be zero because it is possible that some sources of water will not be selected by the optimization model.

$$
F W_{s}^{L}=\min _{p}\left(F P U_{p}^{i n}\right) \quad \forall s \in S W,|p| \neq 0,|s|=1
$$

The upper bound for flowrates going from the initial splitter to the mixer treatment units can be determined on the basis of fix flowrates for all process units.

$$
F I T_{s, t}^{U}=\sum_{p \in P U} F P U_{p}^{i n} \quad \forall s \in S W, \forall t \in T U,|p| \neq 0
$$

In the case that water network consists only of treatment units (number of process units is set to be zero), then wastewater feed flowrates are fixed and the upper bounds of flowrates from the initial splitter to the mixer treatment units and the final mixer are given as follows.

$$
\begin{array}{ll}
F W_{s}^{F X}=F W W_{s}^{i n} & \forall s \in S W,|p|=0 \\
F I T_{s, t}^{U}=F W W_{s}^{i n} & \forall s \in S W, \forall t \in T U,|p|=0 \\
F I F_{s}^{U}=F W W_{s}^{i n} & \forall s \in S W,|p|=0
\end{array}
$$

The upper bound of water flowrate going from the initial splitter to the process units depends of the fixed flowrate in particular process units.

$$
F I P_{s, p}^{U}=F P U_{p}^{i n} \quad \forall s \in S W, \forall p \in P U
$$




\section{Process Units}

The process units can be considered as part of the integrated water network, as well as a separate water-using subsystem. The inlet and outlet flowrates of the process unit are the same. The outlet flowrate of the process unit has fixed value and depends of the inlet flowrate.

$$
F P U_{p}^{\text {out }}{ }^{F X}=F P U_{p}^{\text {in }} \quad \forall p \in P U
$$

The upper and lower bounds for contaminants concentration at the process unit inlets and outlets are given as follows.

$$
\begin{aligned}
& x P U_{p, j}^{i n U}=x P U_{p . j}^{i n, \max } \quad \forall p \in P U, \forall j \\
& x P U_{p, j}^{i n}=10^{3} \cdot L P U_{p, j} / F P U_{p}^{i n} \quad \forall p \in P U, \forall j \\
& x P U_{p, j}^{\text {out } U}=\left(x P U_{p, j}^{i n, \max } \cdot F P U_{p}^{i n}+10^{3} \cdot L P U_{p, j}\right) / F P U_{p}^{i n} \quad \forall p \in P U, \forall j
\end{aligned}
$$

\section{Splitter Process Units}

The splitter process units streams can be directed to any mixer process units so the upper bounds of these flowrates depend of the fixed flowrates in a particular process unit. Notice that the outlet contaminants concentration of the process unit and the splitter process unit are the same.

$$
\begin{array}{lll}
F P_{p^{\prime}, p}^{U}=F P U_{p}^{\text {in }} & \text { if } \quad F P U_{p^{\prime}}^{\text {in }} \leq F P U_{p}^{\text {in }} & \forall p^{\prime} \in P U, \forall p \in P U \\
F P_{p^{\prime}, p}^{U}=F P U_{p}^{i n} \quad \text { if } & F P U_{p^{\prime}}^{\text {in }} \geq F P U_{p}^{\text {in }} & \forall p^{\prime} \in P U, \forall p \in P U \\
x S P U_{p, j}^{\text {out } U}=x P U_{p, j}^{\text {out }} & \forall p \in P U, \forall j &
\end{array}
$$

The upper bounds for flowrates going from the splitter process units to the mixer treatment units and the final mixer depend of the fixed flowrates of process units.

$$
\begin{array}{ll}
F P T_{p, t}^{U}=F P U_{p}^{i n} & \forall p \in P U, \forall t \in T U \\
F P O_{p}^{U}=F P U_{p}^{i n} & \forall p \in P U
\end{array}
$$




\section{Treatment Units}

The treatment units can be considered as part of the integrated water network, as well as a separate wastewater subsystem. According to this, the upper bounds for flowrates and contaminants concentration of the treatment units are given as follows.

$$
\begin{aligned}
& F T U_{t}^{i{ }^{i n}}=\sum_{p \in P U} F P U_{p}^{\text {in }} \quad \forall t \in T U,|p| \neq 0 \\
& F T U_{t}^{\text {in } U}=\sum_{s \in S W} F W W_{s}^{\text {in }} \quad \forall t \in T U,|p|=0 \\
& F T U_{t}^{\text {out } U}=\sum_{p \in P U} F P U_{p}^{\text {in }} \quad \forall t \in T U,|p| \neq 0 \\
& F T U_{t}^{\text {out } U}=\sum_{s \in S W} F W W_{s}^{\text {in }} \quad \forall t \in T U,|p|=0 \\
& x T U_{t, j}^{\text {in } U}=\max _{p \in P U}\left[\left(x P U_{p, j}^{i n, \max } \cdot F P U_{p}^{\text {in }}+10^{3} \cdot L P U_{p, j}\right) / F P U_{p}^{i n}\right] \quad \forall t \in T U, \forall j,|p| \neq 0 \\
& x T U_{t, j}^{\text {in } U}=\max _{s \in S W}\left(x W_{s, j}^{\text {in }}\right) \quad \forall t \in T U, \forall j,|p|=0 \\
& x T U_{t, j}^{\text {out } U}=\left(1-R R_{t, j} / 100\right) \cdot x T U_{t, j}^{\text {in } U}
\end{aligned}
$$

\section{Splitter Treatment Units}

The streams from splitter process units can be directed to any mixer in the network. The upper bounds of these streams are given as follows.

$$
\begin{aligned}
& F T_{t^{\prime}, t}^{U}=\sum_{p \in P U} F P U_{p}^{\text {in }} \quad \forall t^{\prime} \in T U, \forall t \in T U,|p| \neq 0 \\
& F T_{t^{\prime}, t}^{U}=\sum_{s \in S W} F W W_{s}^{\text {in }} \quad \forall t^{\prime} \in T U, \forall t \in T U,|p|=0 \\
& F T P_{t, p}^{U}=F P U_{p}^{\text {in }} \quad \forall t \in T U, \forall p \in P U \\
& F T O_{t}^{U}=\sum_{p \in P U} F P U_{p}^{\text {in }} \quad \forall t \in T U,|p| \neq 0 \\
& F T O_{t}^{U}=\sum_{s \in S W} F W W_{s}^{\text {in }} \\
& x S T U_{t, j}^{\text {out }} U=x T U_{t, j}^{\text {out }} U
\end{aligned}
$$




\section{Source and Demand Units}

It should be noted that inlet and outlet flowrates of water-using operations can have different values. In that case, they can be divided in two parts. The first part can be considered unchanged through process, and the second part can account for the loss of water or the gain of water. According to this, the upper bounds of flowrates and contaminants concentrations are given as follows.

$$
\begin{array}{lc}
F I D_{s, d}^{U}=F D U_{d}^{i n} & \forall s \in S W, \forall d \in D U \\
x D U_{d, j}^{i n^{U}}=x D U_{d, j}^{i n, \max } & \forall d \in D U, \forall j \\
F P D_{p, d}^{U}=F D U_{d}^{i n} & \forall p \in P U, \forall d \in D U \\
F T D_{t, d}^{U}=F D U_{d}^{i n} & \forall t \in T U, \forall d \in D U \\
F S T_{r, t}^{U}=\sum_{p \in P U} F P U_{p}^{i n} & \forall r \in S U, \forall t \in T U \\
F S O_{r}^{U}=F S U_{r}^{\text {out }} & \forall r \in S U \\
F S D_{r, d}^{U}=F D U_{d}^{\text {in }} & \forall r \in S U, \forall d \in D U \\
x S U_{r, j}^{\text {in }}=x S U_{r, j}^{\text {in,max }} & \forall r \in S U, \forall j
\end{array}
$$

\section{Final Mixer}

The lower bound of the discharge flowrate to the environment has a minimum value of the fixed flowrates in the process units. However, if the network consists only of treatment units then it has a minimum value of wastewater streams of the initial splitters.

$$
\begin{array}{ll}
F^{\text {out } L}=\min _{p \in P U}\left(F P U_{p}^{i n}\right) & |p| \neq 0 \\
F^{\text {out } L}=\min _{s \in S W}\left(F W W_{s}^{\text {in }}\right) & |p|=0
\end{array}
$$

The upper bounds of the discharge flowrate and contaminants concentration are given as follows.

$$
F^{\text {out }}{ }^{U}=\sum_{p \in P U} F P U_{p}^{\text {in }} \quad|p| \neq 0
$$




$$
\begin{aligned}
& F^{\text {out }}=\sum_{s \in S W} F W W_{s}^{\text {in }} \quad|p|=0 \\
& x_{j}^{\text {out }}=x_{j}^{\text {out,max }} \quad \forall j \\
& x_{j}^{\text {out }}=\max _{p \in P U}\left[\left(x P U_{p, j}^{\text {in, } \text { max }} \cdot F P U_{p}^{\text {in }}+10^{3} \cdot L P U_{p, j}\right) / F P U_{p}^{\text {in }}\right] \quad \forall j,|t|=0
\end{aligned}
$$

\section{References}

Alva-Argaez, A., Kokossis, A. C. \& Smith, R. (2007). The design of water-using systems in petroleum refining using a water-pinch decomposition. Chemical Engineering Journal, 128(1), 33-46.

Alva-Argáez, A., Kokossis, A. C. \& Smith, R. (1998). Wastewater minimisation of industrial systems using an integrated approach. Computers \& Chemical Engineering, 22(Supplement 1), S741-S744.

Bagajewicz, M. (2000). A review of recent design procedures for water networks in refineries and process plants. Computers \& Chemical Engineering, 24(9-10), 2093-2113.

Bagajewicz, J. \& Faria D. C. (2009). On the appropriate architecture of the water/wastewater allocation problem in process plants. In J. Ježovski \& J. Thullie (Editors), $19^{\text {th }}$ European Symposium on Computer Aided Process Engineering - ESCAPE19. Elsevier.

Bandyopadhyay, S., Ghanekar, M. D. \& Pillai, H. K. (2006). Process water management. Industrial \& Engineering Chemistry Research, 45(15), 5287-5297.

Biegler, L. T., Grossmann, I. E. \& Westerberg, A. W. (1997). Systematic methods of chemical process design. New Jersey, USA: Prentice-Hall.

Brooke, A., Kendrick, D., Meeraus, A. \& Raman, R. (1988). GAMS: A user's guide, release 2.50. GAMS Development Corporation.

Castro, P., Matos, H., Fernandes, M. C. \& Nunes, C. P. (1999). Improvements for massexchange networks design. Chemical Engineering Science, 54(11), 1649-1665.

Castro, P., Teles, J. P. \& Novais, A. (2009). Linear program-based algorithm for the optimal design of wastewater treatment systems. Clean Technologies and Environmental Policy, 11(1), 83-93.

Dhole, V. R., Ramchandani, N., Tainsh, R. A. \& Wasilewski, M. (1996). Make Your Process Water Pay for Itself. Chemical Engineering, 103, 100. 
Doyle, S. J. \& Smith, R. (1997). Targeting water reuse with multiple contaminants. Process Safety and Environmental Protection, 75(3), 181-189.

El-Halwagi, M. M. (1997). Pollution prevention through process integration. Systematic design tools. San Diego, USA: Academic Press.

El-Halwagi, M. M. \& Manousiouthakis, V. (1989). Synthesis of mass exchange networks. AIChE Journal, 35(8), 1233-1244.

El-Halwagi, M. M. \& Manousiouthakis, V. (1990). Automatic synthesis of mass-exchange networks with single-component targets. Chemical Engineering Science, 45(9), 2813-2831.

Faria D. C. \& Bagajewicz, J. (2009). On the appropriate modeling of process plant water systems. (http://www.ou.edu/class/che-design/).

Feng, X. \& Seider, W. D. (2001). New structure anddesign methodology for water networks. Industrial \& Engineering Chemistry Research, 40(26), 6140-6146.

Foo, D. C. Y., Kazantzi, V., El-Halwagi, M. M. \& Abdul Manan, Z. (2006). Surplus diagram and cascade analysis technique for targeting property-based material reuse network. Chemical Engineering Science, 61(8), 2626-2642.

Foo, D. C. Y. (2009). State-of-the-Art Review of Pinch Analysis Techniques for Water Network Synthesis. Industrial \& Engineering Chemistry Research, 489(11), 5125-5159.

Galan, B. \& Grossmann, I. E. (1998). Optimal design of distributed wastewater treatment networks. Industrial \& Engineering Chemistry Research, 37(10), 4036-4048.

Gunaratnam, M., Alva-Argaez, A., Kokossis, A., Kim, J.-K. \& Smith, R. (2005). Automated Design of Total Water Systems. Industrial \& Engineering Chemistry Research, 44(3), 588599.

Hallale, N. (2002). A new graphical targeting method for water minimisation. Advances in Environmental Research, 6(3), 377-390.

Huang, C.-H., Chang, C.-T. Ling, H.-C. \& Chang, C.-C. (1999). A Mathematical Programming Model for Water Usage and Treatment Network Design. Industrial \& Engineering Chemistry Research, 38(8), 3190-3190.

Ježowski, J. (2008). Review and analysis of approaches for designing optimum industrial water networks. Chemical and Process Engineering, 29, 663-681.

Karuppiah, R. \& Grossmann, I. E. (2006). Global optimization for the synthesis of integrated water systems in chemical processes. Computers \& Chemical Engineering, 30(4), 650-673. 
Karuppiah, R. \& Grossmann, I. E. (2008). Global optimization of multiscenario mixed integer nonlinear programming models arising in the synthesis of integrated water networks under uncertainty. Computers \& Chemical Engineering, 32(1-2), 145-160.

Kuo, W.C. J. \& Smith, R. (1997). Effluent treatment system design. Chemical Engineering Science, 52(23), 4273-4290.

Li, B.-H. \& Chang, C.-T. (2007). A Simple and Efficient Initialization Strategy for Optimizing Water-Using Network Designs. Industrial \& Engineering Chemistry Research, 46(25), 87818786.

Linnhoff, B. \& Hindmarsh, E (1983). The pinch design method for heat exchanger networks. Chemical Engineering Science, 38(5), 745-763.

LINDOGlobal: A Global Optimization Solver from Lindo Systems, Inc.

Luo, Y. \& Uan, X (2008). Global Optimization for the Synthesis of Integrated Water Systems with Particle Swarm Optimization Algorithm. Chinese Journal of Chemical Engineering, 16(1),11-15.

Mann, J. G. \& Liu, Y. A. (1999). Industrial water reuse and wastewater minimization. New York, USA: McGraw-Hill.

Polley, G. T. \& Polley, H. L. (2000). Design Better Water Networks. Chem. Eng. Progress, 96, 47.

Putra, Z. A. \& Amminudin, K. A. (2008). Two-Step Optimization Approach for Design of A Total Water System. Industrial \& Engineering Chemistry Research, 47(16), 6045-6057.

Quesada, I. \& Grossmann, I. E. (1995). Global optimization of bilinear process networks with multicomponent flows. Computers \& Chemical Engineering, 19(12), 1219-1242.

Rossiter, A. P. (1995). Waste minimization through process design. New York, USA: McGrawHill.

Sahinidis, N. V. (1996). BARON: A general purpose global optimization software package. Journal of Global Optimization 8(2): 201-205.

Savelski, M. \& Bagajewicz, M. (2003). On the necessary conditions of optimality of water utilization systems in process plants with multiple contaminants. Chemical Engineering Science, 58(23-24), 5349-5362. 
Savelski, M. J. \& Bagajewicz, M. J. (2000). On the optimality conditions of water utilization systems in process plants with single contaminants. Chemical Engineering Science, 55(21), 5035-5048.

Sorin, M. \& Bedard, S. (1999). The global pinch point in water reuse networks. Process Safety and Environmental Protection, 77(B5), 305-308.

Takama, N., Kuriyama, T., Shiroko, K. \& Umeda, T. (1980). Optimal water allocation in a petroleum refinery. Computers \& Chemical Engineering, 4(4), 251-258.

Wang, Y. P. \& Smith, R. (1994a). Wastewater minimisation. Chemical Engineering Science, 49(7), 981-1006.

Wang, Y.P. \& Smith, R. (1994b). Design of distributed effluent treatment systems. Chemical Engineering Science, 49(18), 3127-3145.

Wang, Y. P. \& Smith, R. (1995). Wastewater Minimization with Flowrate Constraints. Chem. Eng. Res. Des., 73, 889. 